\title{
Experimental investigation of the re-centring capability of Curved Surface Sliders
}

\begin{tabular}{|r|l|}
\hline Journal: & Structural Control and Health Monitoring \\
\hline Manuscript ID & STC-15-0272.R1 \\
\hline Wiley - Manuscript type: & Research Paper \\
\hline Date Submitted by the Author: & n/a \\
\hline Complete List of Authors: & $\begin{array}{l}\text { Quaglini, Virginio; Politecnico di Milano, Department of Architecture, Built } \\
\text { Environment and Construction Engineering } \\
\text { Gandelli, Emanuele; Politecnico di Milano, Department of Architecture, Built } \\
\text { Environment and Costruction Engineering } \\
\text { Dubini, Paolo; Politecnico di Milano, Department of Architecture, Built } \\
\text { Environment and Costruction Engineering }\end{array}$ \\
\hline Keywords: & $\begin{array}{l}\text { base isolation, curved surface sliders, re-centring capability, shake-table } \\
\text { test, displacement accrual, residual displacement }\end{array}$ \\
\hline &
\end{tabular}




\title{
Experimental investigation of the re-centring capability of Curved Surface Sliders
}

\author{
Virginio Quaglini ${ }^{(*)}, \mathrm{PhD}$, Emanuele Gandelli, MSc, Paolo Dubini, PhD \\ Politecnico di Milano, Department of Architecture, Built Environment and Construction \\ Engineering. Piazza Leonardo da Vinci 32, 20133 Milano \\ ${ }^{(*)}$ corresponding author \\ Email: virginio.quaglini@polimi.it \\ Phone: +39 02.2399.4248
}




\section{ABSTRACT}

The re-centring capability is recognized as a fundamental function of the isolation system, since it is intended to prevent substantial permanent deformation at the end of the earthquake that may affect the serviceability of the structure and eventually limit the capability of the isolators to withstand aftershocks and future earthquakes. In this study the re-centring behaviour of isolation systems comprised of sliding bearings with curved surfaces is investigated in shake table tests carried out on a one-story steel frame with rectangular plan, scaled at one third-length scale and isolated with four bearings. The coefficient of friction of the bearings is varied by changing the material or lubrication condition of the pads, providing different equivalent damping ratios to the isolation system. The response of the base isolated structure to selected natural ground motion waveforms is assessed in terms of the residual displacement after a single event and the accrual of displacements during a sequence of quakes, and considerations on the influence of the coefficient of friction on the recentring behaviour, as well as on the effect of an initial displacement offset are drawn. The recentring provision of the current European design code is eventually checked against the experimental data.

Keywords: base isolation; curved surface sliders; re-centring capability; shake-table test; displacement accrual; residual displacement 


\section{INTRODUCTION}

Base isolation is a modern strategy for seismic protection based on the provision of the construction with a set of bearings that decouple the motion of the superstructure from the motion of the ground. The bearings are aimed at modifying the response of the superstructure by lengthening its fundamental period, which reduces forces but increases displacements, and by increasing damping, which reduces displacements and may reduce forces.

A fundamental function of the base isolation system is recognized to be the ability to return towards the origin at the end of ground motions or tremors, or self re-centring [1-3]. This function is associated with the prevention of large residual displacement after the earthquake, which may affect the serviceability of the structure and jeopardize the functionality of elements crossing the isolation plane (like fire protection and weather proofing elements, egress/entrance details, elevators, joints of primary piping systems etc.), and eventually limit the capability of the isolation system to withstand aftershocks and future earthquakes [4]. There is significant field evidence of seismic sequences characterized by frequent medium-strong intensity ground motions following a strong mainshock after short intervals of time [5-10]. Since it may not be possible to re-center the system before close aftershocks, ground motion sequences with such characteristics would entail an accrual of displacements, and the deformation capacity of the isolation system designed based on a single earthquake possibly become inadequate.

In the past, structural engineers did not pay much attention to the re-centring capability of seismic isolators, and the first studies focused on the residual displacements of generic low-ductility nonlinear SDOF systems [11-14]. This was probably a consequence of the fact that the first seismic isolators were conventional laminated rubber bearings that were endowed with an inherent selfcentring capability associated with the elastic restoring force developed in the rubber layers [15-16]. However in the recent years, with the introduction of new isolation devices with high energy dissipation capacity like lead rubber bearings, sliding isolators with steel hysteretic elements, friction 
bearings, etc., the problem of limiting residual displacements turned out of primary interest. Criteria for evaluating the re-centring capability of the isolation system have been provided in modern seismic codes. According to the European code, or Eurocode 8 [17], this capability is available in one horizontal direction when the system has small residual displacements in relation to its displacement capacity, and the requirement is considered satisfied when the condition is met:

$$
\frac{d_{c d}}{d_{0}} \geq \delta
$$

where $d_{c d}$ is the design displacement of the isolation system in the examined direction, $d_{0}$ is the "maximum static residual displacement" for which the isolation system can be in static equilibrium in the considered direction, i.e. the residual displacement under which the static equilibrium is reached at unloading from $d_{c d}$ under quasi-static conditions (Figure 1), and $\delta$ is a numeric coefficient, whose recommended value is 0.5 .

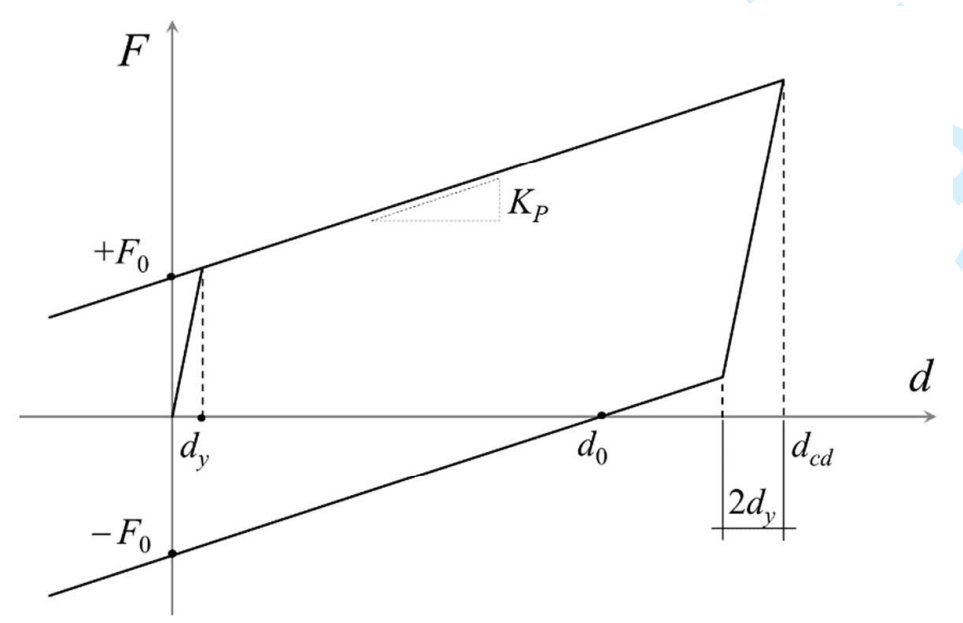

Figure 1. Equivalent bilinear hysteretic model of the isolation system for the evaluation of the re-centring capability

The validity of this criterion for isolation systems with the typical bilinear hysteretic behaviour shown in Figure 1 was assessed in a parametric study [3], analysing 150 different combinations of system parameters and 122 natural ground motions. Systems with $d_{c d} / d_{0} \geq 0.5$ demonstrated small 
residual displacements compared with the maximum displacement induced by the ground motion, and insignificant accumulation of residual displacements at the end of a sequence of earthquakes. Similarly, an experimental study on isolation systems for bridges comprised of flat sliding bearings, rubber devices and fluid dampers [15] showed that the a strong re-centring capability is provided when the ratio of the characteristic strength $F_{0}$ to the peak restoring force at the maximum displacement $d_{\max }$, i.e. $K_{P} d_{\max }$, is less than or equal to 3 , which corresponds to $d_{\max } / d_{0} \geq 0.3$ ( $F_{0}$ and $K_{P}$ defined as in Figure 1).

The European design code requires that systems that do not satisfy the re-centring provision of Equation (1), have anyway sufficient displacement capacity to accommodate, with adequate reliability, the accrual of residual displacements during the service life of the structure. To account for the possible displacement build-up under a sequence of earthquake events occurring before the design earthquake,-the displacement demand must be increased by a factor

$$
\rho_{d}=1+1.35 \frac{1-\left(d_{y} / d_{c d}\right)^{0.6}}{1+80\left(d_{c d} / d_{0}\right)^{1.5}}
$$

where $d_{y}$ is the yield displacement (see Figure 1) of the equivalent bilinear system. For systems with $d_{c d} / d_{0}>0.5, \rho_{d}<1.05$ and the effect of the accumulation of residual displacements is insignificant.

A second re-centring criterion, based on energy concepts, is provided in the European standard on antiseismic devices EN 15129 [18]: the system has sufficient re-centring capability when the relation is met

$$
E_{S} \geq 0.25 E_{H}
$$

where $E_{S}$ is the reversibly stored elastic energy and $E_{H}$ is the energy dissipated in hysteretic deformation when the isolation system moves from its origin to the position of maximum displacement. It can be easily demonstrated that for bilinear hysteretic systems the provision of Equation (3) is identical to Equation (1) where $\delta=0.5$. 
Among the most popular antiseismic hardware today there is the Curved Surface Slider (CSS), which was firstly introduced in North America in the second part of the ' 90 s in the version called the Friction Pendulum System ${ }^{\circledR}$, or FPS $^{\circledR}$ [19-21], and whose wide use is in progress in Europe and worldwide after the expiry of patent rights in 2005. The Curved Surface Slider is based on the principle of accommodating the horizontal displacement of the structure by means of one of more pair(s) of curved sliding surfaces, which provide a restoring force due to the effect of the curvature and dissipate seismic energy through friction forces developed during sliding. Energy dissipation and self-centring capability are two antithetic functions, as friction forces can act away from the origin contrasting the effect of the restoring force [1], and a potential issue that has come recently under attention is the occurrence of large residual displacements when materials with high levels of friction are used in the sliding surfaces. This concern is further of particular importance when the sliding material exhibits the so-called "stick-slip" phenomenon, i.e. a continuous transition from the sliding phase to the sticking phase and vice versa occurring at slow velocities [22]: in the sticking phase the coefficient of friction rises from its dynamic (or kinetic) level to the static level hence increasing the resistance to the re-centring motion of the isolating system.

In the light of these considerations, it is therefore questionable whether the re-centring criterion of the Eurocode 8 is valid for Curved Surface Sliders. The criterion was indeed formulated under the assumption that the mechanical properties of the isolation system, lumped in the parameter $d_{0}$ in Equation (1), are constant. This is in general not true for the CSS, as the coefficient of friction is noted to be dependent on a number of factors among which there are the axial load, the velocity of sliding and the temperature [23-27]. Research addressing the re-centring capability of Curved Surface Sliders is very recent, and definite results have not yet been achieved. A parametric study [28]pointed that the code's provision seems to be not conservative and sliding isolators with curved surfaces experience negligible residual displacements only when $d_{c d} / d_{0}$ is larger than 2.5 . Also the experimental knowledge is little. Shake table tests on a 3-story seismically isolated structure 
equipped with triple friction pendulum isolators and simulating sequences of low-to-moderate and high amplitude seismic ground motions, concluded that depending on the sequence details, the isolation system may either accumulate or recover residual displacements [29].

The evaluation of the self-centring capability of the Curved Surface Slider is of primary importance since it can significantly affect the design of the isolation system, as well as of the isolated structure that must be designed to accommodate the residual displacements. The present study aims at giving an insight into the matter, providing the results of an experimental investigation conducted within the framework of the Task 6 "Isolation and Dissipation" of the Italian RELUIS (National Network of Laboratories of Seismic Engineering) Project 2010-2013. The two main goals are: (1) to evaluate the re-centring capability of curved surface sliders comparing devices with different coefficient of friction, and (2) to assess the influence of permanent displacements on the amplitude of the seismically induced motion and the possible accrual of displacements. 


\section{Theoretical considerations}

The force - displacement behaviour of the Curved Surface Slider is conventionally described [30] in accordance with the bilinear hysteretic model illustrated in Figure 1 , where $F_{0}$ is the characteristic strength and $K_{P}$ is the restoring stiffness of the isolator. Both properties depend on the characteristics of the curved surfaces according to the relations $F_{0}=\mu_{d} \cdot N$ and $K_{P}=N / R_{b}$ where $N$ is the vertical load acting through the bearing, $\mu_{d}$ is the dynamic coefficient of friction, and $R_{b}$ is the effective radius of curvature [30]. For the Curved Surface Slider the yield displacement $d_{y}$ is usually negligible and can be assumed to be zero [17]. The horizontal reaction force $F$ of the isolator at a displacement $d$ is therefore given by:

$$
F=F_{0} \cdot \operatorname{sign}(\dot{d})+K_{P} \cdot d
$$

where $\operatorname{sign}(\dot{d})$ is the sign of the velocity vector.

The static equilibrium between the restoring force $K_{P} \cdot d$ that always acts towards the origin, and the friction force $\mu_{d} \cdot N$ that contrasts the motion is attained at the displacement $d_{0}$

$$
d_{0}=\frac{F_{0}}{K_{P}}=\mu_{d} \cdot R_{b}
$$

which represents the upper bound to the actual residual displacement $d_{r}$ at the end of the motion and is independent on the displacement capacity of the isolator. Assuming for simplicity that the motion of the bearing is symmetric with respect to the origin, with amplitude equal to $d_{c d}$, then the effective damping can be expressed as

$$
\xi_{b}=\frac{2}{\pi} \cdot \frac{\mu_{d} \cdot R_{b}}{d_{c d}}=\frac{2}{\pi} \cdot \frac{d_{0}}{d_{c d}}
$$

Equation (6) confirms that increasing the energy dissipation capacity of the bearing entails a corresponding decrease in the re-centring capability expressed by the ratio $d_{c d} / d_{0}$.

For the Curved Surface Slider the provisions (1) and (3) can be reformulated as [31] 


$$
\frac{d_{c d}}{\mu_{d} \cdot R_{b}} \geq 0.5
$$

The dependency of the coefficient of friction on the sliding velocity has a fundamental importance in the response of the isolation system during the earthquake ground motion. According to a widely recognized model [23], this dependency is expressed through the exponential law

$$
\mu_{d}=\mu_{\text {fast }}-\left(\mu_{\text {fast }}-\mu_{\text {slow }}\right) \cdot \exp (-\alpha \cdot V)
$$

where $\mu_{\text {slow }}$ and $\mu_{\text {fast }}$ are the levels of the friction coefficient at low and fast velocities, respectively, $V$ is the sliding velocity and $\alpha$ is a rate parameter, with dimensions of the inverse of velocity, which depends on axial load and air temperature [24]. The coefficient of friction increases more than linearly with increasing the velocity, and the value of $\mu_{\text {fast }}$ is important in determining the maximum displacement of the isolation system that is achieved during the strong motion stage of the earthquake when the system absorbs energy. On the contrary, a lower level of friction governs the response of the system during the coda stage of the earthquake, when the seismic energy input can be considered as insignificant. Therefore, it is reasonable to assume the value $\mu_{d}=\mu_{\text {slow }}$ in Equation (5) wherein the force equilibrium under quasi-static condition is considered:

$$
d_{0}=\mu_{\text {slow }} \cdot R_{b}
$$

A possible outcome of an insufficient re-centring capability is the increase in residual and maximum displacements for ground motions with directivity effects, like near-fault earthquakes, where the most of the energy is introduced in the system by a single pulse [2; 32]. For ground motions with such characteristic, the effect of a displacement offset is now examined using energy concepts. The movement of the isolation system from its origin to the maximum displacement $d_{\max }$ is represented in Figure 2 as a solid line (branch OAB). Assume now that at the beginning of the earthquake the system is affected by the eccentricity $\Delta d_{i}=$ OO', which could be due to e.g. the residual displacement at the end of a preshock. The offset leads to a corresponding change $\Delta d_{\max }$ in the maximum displacement (branch O'A'B'). As the energy induced by the earthquake is the same for 
the two examined branches, the law of conservation of energy requires the two shaded areas $E_{1}$ and $E_{2}$ to be equal. This implies that the two quantities $\Delta d_{i}$ and $\Delta d_{\max }$ have the same sign and the relation between them is provided by simple geometrical calculations [3]

$$
\Delta d_{\max }=\frac{1}{1+\frac{d_{\max }}{d_{0}}} \Delta d_{i}
$$

In Equation (10) the sign of $\Delta d_{i}$ (and consequently of $\Delta d_{m a x}$ ) is positive when the offset is in the same direction along which the maximum displacement $d_{\max }$ occurs, and negative otherwise (Figures 2(a) and 2(b) respectively). The displacement accrual depends on both the properties of the isolators, expressed by the parameter $d_{0}$, and the details of the ground motion, which affect the magnitude and the direction of the peak displacement $d_{\max }$. As evident from Equation (10), the influence of the offset is expected to be lower for strong earthquakes producing larger motions than for weak earthquakes.

However, it has to be noted that a decrease of the maximum displacement may be also possible. When the isolation is in its origin (position $\mathrm{O}$ ), the inertial force produced by the ground acceleration must overcome the characteristic strength $F_{0}$ before the system is displaced, and owing to the symmetry of the loop (points A and E in Figure 2(a)), the motion has the same likelihood of starting in either direction. On the contrary, when the offset $\Delta d_{i}$ is present, the breakaway resistance is no longer symmetric since the restoring force is always directed towards the origin: the resisting force is (in its absolute value) equal to $F_{0}+K_{\mathrm{P}} \cdot \Delta d_{i}$ for motion in the positive direction of the displacement axis (point $\mathrm{A}^{\prime}$ ), and equal to $F_{0}-K_{\mathrm{P}} \cdot \Delta d_{i}$ (point E') for motion in the opposite direction. Hence, a lower magnitude of ground acceleration will be enough to initiate the motion in the direction opposite to the offset than in the same direction, which entails a decrease in $d_{\max }$ according to Equation (10). Similar conclusions apply to the situation of Figure 2(b), with consideration of the different sign. 
In the above considerations, the dependency of the coefficient of friction on the axial load and on the temperature of the sliding surfaces was not accounted for. In practice, the axial load acting on the individual bearings can undergo considerable variations during the earthquake due to the rocking of the superstructure and the vertical ground excitation, and heating of the sliding surfaces occurs as well following the huge dissipation of seismic energy by means of friction. All these effects contribute to changes in the actual coefficient of friction of the CSS surfaces in addition to the effect of velocity. Though the rigorous conclusion were that the classic bilinear model is not representative of the actual behaviour of the Curved Surface Slider under seismic excitation, it is of practical interest to verify whether the conclusions drawn for the simple bilinear systems are still applicable, at some extent, to describe the basic features of the re-centring behaviour of CSS systems.

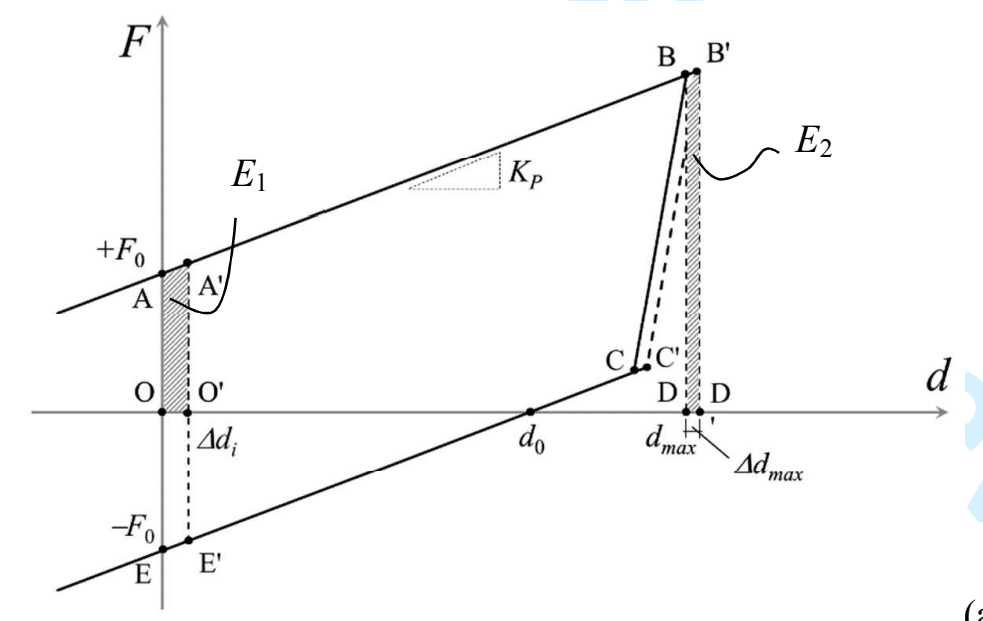

(a)

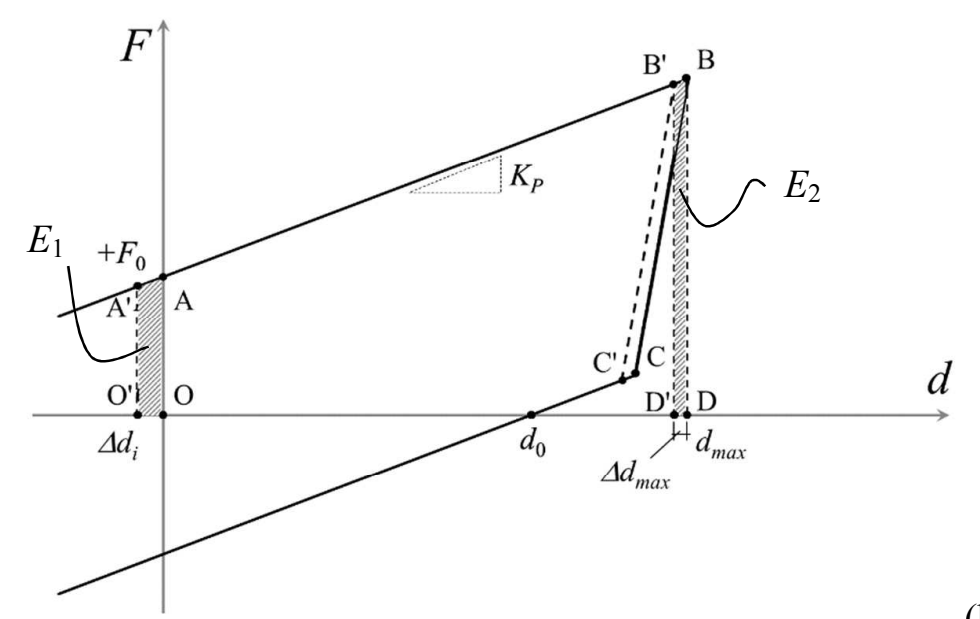


1

2

3

4

5

6

7

8

9

10

11

12

13

14

15

16

17

18

19

20

21

22

23

24

25

26

27

28

29

30

31

32

33

34

35

36

37

38

39

40

41

42

43

44

45

46

47

48

49

50

51

52

53

54

55

56

57

58

59

60
Figure 2. Influence of the initial offset $\Delta d_{i}$ on the maximum displacement $d_{\max }: \Delta d_{i}>0$ and $\Delta d_{\max }>0$ (a); $\Delta d_{i}<0$ and $\Delta d_{\max }<0$ (b) 


\section{MATERIALS AND METHODS}

\section{Experimental set-up}

The investigation was performed at the laboratory of the Department of Structures for Engineering and Architecture at the University "Federico II" of Naples, Italy. The testing facility was a $3 \times 3 \mathrm{~m}$ biaxial shake table, with maximum payload of $200 \mathrm{kN}$, frequency range of $0-50 \mathrm{~Hz}$, acceleration peak equal to $1 \mathrm{~g}$, velocity peak equal to $1 \mathrm{~m} / \mathrm{s}$ and total displacement capacity of 500 $\mathrm{mm}$.

The mock-up consisted of a four columns, one story steel frame with a rectangular plant of $2.65 \times 2.15 \mathrm{~m}$ span and $2.90 \mathrm{~m}$ total height (Figure 3 ), which could be assumed as representative of a building at one third-length scale. Details of the mock-up can be found elsewhere [33]. Each floor contained additional concrete blocks / slabs to provide a total mass of 8200 tons. The base level has a mass of 3.26 tons, whereas the top floor had a mass of 4.94 tons.

The mock-up was supported by four bearings bolted to the base frame in correspondence of the columns. Owing to the symmetry of the structure, each isolator carried a quarter of the total weight. In order to prevent the structure from dropping off the shake table in case of excessive movements, safety constraints limited the relative displacements to $\pm 125 \mathrm{~mm}$. 


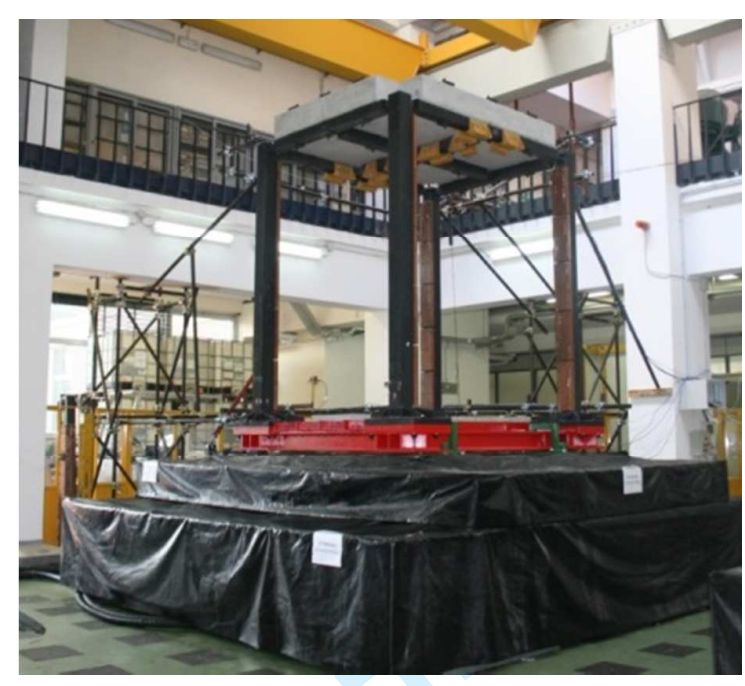

(a)

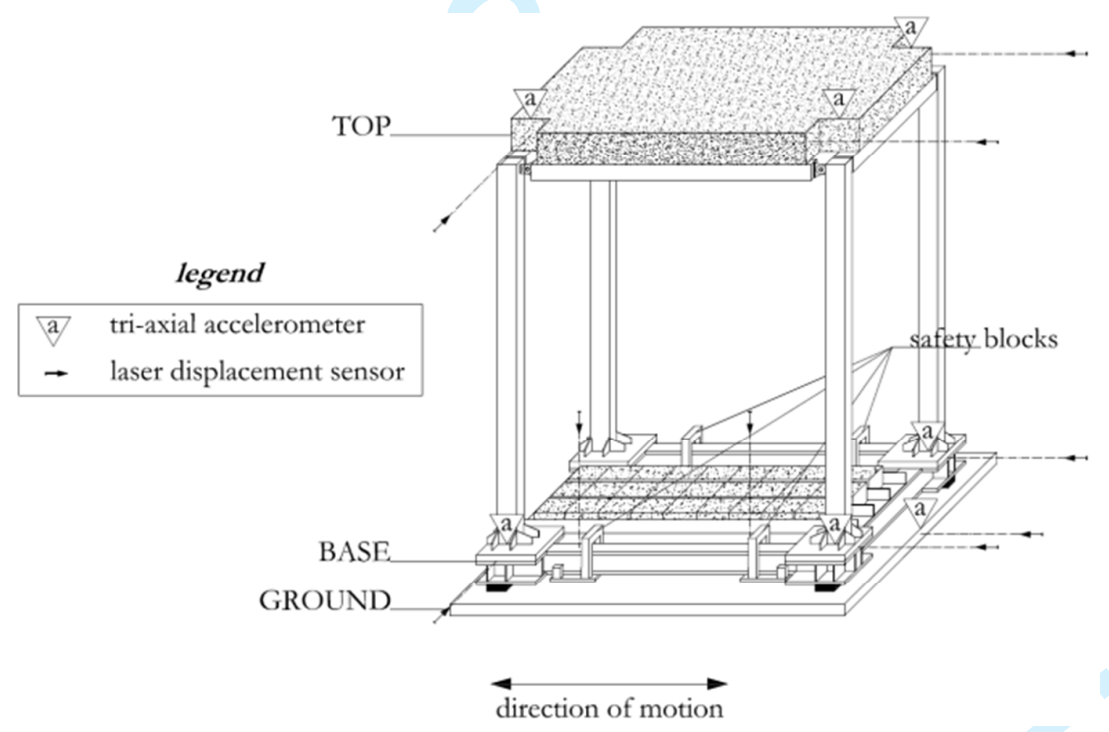

(b)

Figure 3. Test set-up: view of the structure mock-up on the shake table (a); and cabinet projection of the mock-up and the instrumentation set-up (b).

The scale factors of the structural model are summarized in Table 1. The fundamental frequency of the fixed-base model is $2.5 \mathrm{~Hz}$ (i.e. a period $\mathrm{T}=0.4 \mathrm{~s}$ ), which corresponds to a period of the fullscale structure of $0.7 \mathrm{~s}$, common for seven-story buildings. The damping for the fundamental mode is $0.6 \%$, similar to the majority of steel frames in the elastic range with no additional sources of energy dissipation. 
1

2

3

4

5

6

8

10

In the experiments, unidirectional acceleration time histories were imposed to the shake table along the direction of maximum span of the mock-up. The absolute displacements of the mock up were monitored by six laser displacement sensors, model CP35MHT80 (Wenglor Sensoric GmbH, Germany), with $\pm 150 \mathrm{~mm}$ capacity and $50 \mu \mathrm{m}$ resolution; at each floor two transducers were used to measure the displacement in the direction of motion, whereas one transducer measured the displacement in the transverse direction for detecting any in-plane rotations. The absolute accelerations of the floor masses were measured by means of six triaxial accelerometers model 356A17 (PCB Piezotronics Inc, NY), with \pm 10 g capacity, placed at the corners of structure, three at each floor. To complete the instrumentation layout, an additional laser transducer was used to measure the absolute displacement of the table in the direction of testing, and an accelerometer recorded the motion of the table to check the actual ground motion transmitted to the structure. Displacements and accelerations measured by the sensors were sampled at $500 \mathrm{~Hz}$ and filtered at 50 Hz. The instrumentation set-up is illustrated in Figure 3(b). 
Table 1. Summary of Scale Factors.

\begin{tabular}{llc}
\hline Variable & Dimension & Scale Factor \\
\hline Linear Dimension & Length & $1 / 3$ \\
Displacement & Length & $1 / 3$ \\
Time & Time & $1 / \sqrt{ } 3$ \\
Mass & Mass & $1 / 9$ \\
Velocity & Length $\cdot$ Time $^{-1}$ & $1 / \sqrt{ } 3$ \\
Acceleration & Length· Time & 1 \\
Frequency & Time $^{-2}$ & $\sqrt{3}$ \\
Force & ${\text { Mass } \cdot \text { Length } \cdot \text { Time }^{-2}}$ & $1 / 9$ \\
\hline
\end{tabular}

\section{Curved Surface Sliders}

Curved Surface Slider prototypes were manufactured for the $1 / 3$ scaled model according to two alternative designs depending on the displacement capacity but with same effective radius and restoring stiffness. The first design was a Double Curved Surface Slider (DCSS), Figure 4(a), with upper and lower concave backing plates having identical radii $R_{1}=R_{2}=770 \mathrm{~mm}$, and a rigid slider with height $h=55 \mathrm{~mm}$, providing an effective radius $R_{b}=R_{1}+R_{2}-h=1485 \mathrm{~mm}$ [34]; the displacement capacity was $260 \mathrm{~mm}$. The second design was a Single Curved Surface Slider (SCSS), Figure 4(b), with radii $R_{1}=1270 \mathrm{~mm}$ and $R_{2}=300 \mathrm{~mm}$, respectively, and a rigid slider with height $h=70 \mathrm{~mm}$, providing an effective radius $R_{b}=1500 \mathrm{~mm}$; the displacement capacity was $165 \mathrm{~mm}$.

Four SCSS bearings and four DCSS bearings were produced and assembled. The backing plates and the sliders of the bearings were made of S355 structural steel, with the concave sliding surfaces lined with a $2.5 \mathrm{~mm}$ thick stainless steel sheet. The sliding pads had a diameter of either $80 \mathrm{~mm}$ (SCSS bearing) or $60 \mathrm{~mm}$ (DCSS bearing) and a thickness of $6 \mathrm{~mm}$. A rotation pad made of 
lubricated PTFE was used in the spherical joint (curved surfaces with radius $R_{2}$ ) of the SCSS bearings.

Three different materials were used for the sliding pads:

- standard PTFE (polytetrafluoroethylene) resing lubricated with silicon grease (L-TF);

- composite material made of PTFE resin with metal fillers, lubricated with silicon grease (L-CM);

- composite material made of PTFE resin with metal fillers, not lubricated (NL-CM).

L-TF and L-CM pads were used in the DCSS bearings, whereas NL-CM pads in the SCSS bearings.

The coefficient of friction of the three materials material was characterised in small scale tests conducted at the laboratory of Politecnico di Milano in accordance with the experimental procedure described elsewhere [25]. The material specimens were tested under the application of a constant pressure of either $7.10 \mathrm{MPa}$ (L-TF and L-CM materials) or $3.98 \mathrm{MPa}$ (NL-CM material), corresponding to the actual load acting on the pads when installed the CSS bearings due to the weight of the mock-up. The experimental data obtained in the tests were curve-fitted in accordance with Equation (8), providing the values of the $\mu_{\text {slow }}, \mu_{\text {fast }}$ and $\alpha$ parameters reported in Table 2.

Table 2. Parameters of the friction law of the pad materials and relevant test pressure $p$ (ambient temperature, velocity range: 1 to $200 \mathrm{~mm} / \mathrm{s})$.

\begin{tabular}{ccccc}
\hline Material & pressure, MPa & $\mu_{\text {slow }}$ & $\mu_{\text {high }}$ & $\alpha, \mathrm{s} / \mathrm{mm}$ \\
\hline L-TF & 7.10 & 0.008 & 0.039 & 0.003 \\
L-CM & 7.10 & 0.022 & 0.100 & 0.002 \\
NL-CM & 3.98 & 0.032 & 0.265 & 0.002 \\
\hline
\end{tabular}




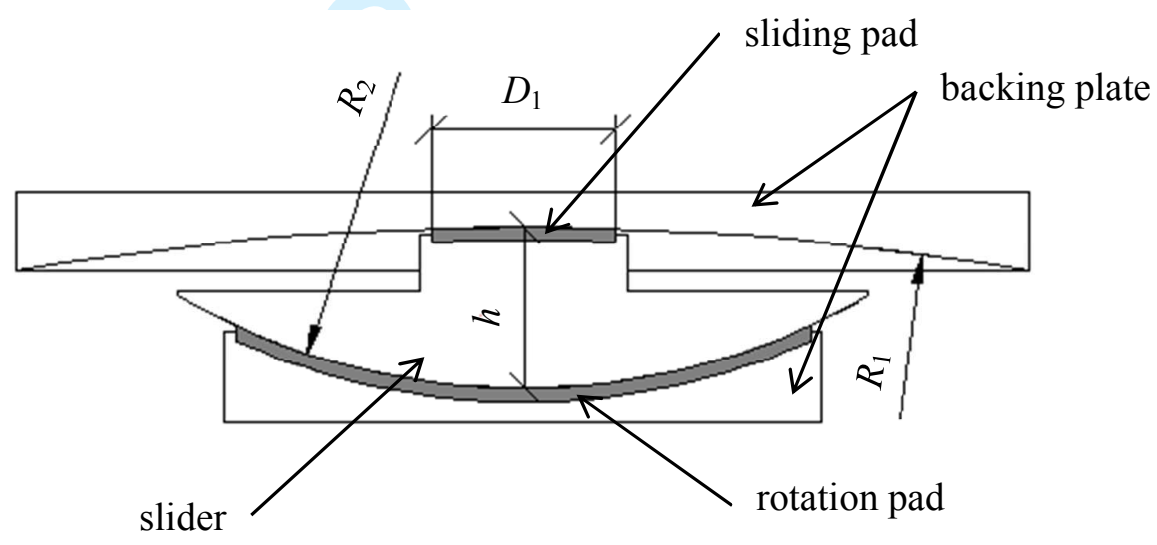

Figure 4. Geometrical parameters of Double Curved Surface Slider (DCSS) prototype (a), and Single Curved Surface Slider (SCSS) prototype (b)

\section{Characterization of the isolation system}

Preliminary to the seismic tests, the dynamic properties of the isolation system were characterized by means of sine sweep excitation tests conducted on the isolated mock-up. A displacement amplitude of $50 \mathrm{~mm}$ was imposed to the table motion with frequency varying from 0.25 to $1.5 \mathrm{~Hz}$ with an incremental step of $0.05 \mathrm{~Hz}$. Three displacement cycles at every frequency level were carried out. Table 3 reports for the different configurations the fundamental frequency $f_{\text {iso }}$ of the base isolated structure obtained by Fast Fourier Transforms (FFTs) of the roof acceleration signals, the corresponding fundamental period $T_{\text {iso }}$, and the properties of the isolation system at $f_{\text {iso }}$. 
Table 3. Fundamental properties of the isolation system and corresponding dynamic properties.

\begin{tabular}{lllllll}
\hline Bearing model & Sliding pad & $f_{\text {iso }}, \mathrm{Hz}$ & $T_{\text {iso }}, \mathrm{s}$ & $\xi_{\text {eq }},-$ & $F_{0}, \mathrm{kN}$ & $K_{P}, \mathrm{kN} / \mathrm{m}$ \\
\hline DCSS & L-TF & 0.55 & 1.82 & 28.5 & 0.603 & 13.54 \\
DCSS & L-CM & 1.06 & 0.94 & 54.2 & 1.608 & 13.54 \\
SCSS & NL-CM & 1.16 & 0.86 & 55.8 & 2.412 & 13.41 \\
& & & & & & \\
\hline
\end{tabular}

\section{Ground motion records}

Seven independent ground motion records consistent with the current Italian Building Code [35] were selected from the European Strong-motion Database [36] using REXEL v3.4 beta software [37]. The selected horizontal acceleration histories are in compliance with the assumed code for the life safety limit state of a strategic structure (functional class IV) located in Naples, Italy (14.2767 longitude, $40.863^{\circ}$ latitude) on soil type A (stiff soil or rock) with a nominal life of 100 years (corresponding to a 1898-year return period according to the code). Only events in the magnitude $(\mathrm{Mw})$ interval $[5.3,7.3]$ and with an epicentral distance $(R)$ interval $[0-80 \mathrm{~km}]$ were considered, which reflects the hazard disaggregation for the spectral acceleration $\mathrm{Sa}(\mathrm{T})$ for the period of interest in the nonlinear structural behaviour. This selection is representative of regions in Italy with a moderate to high seismic risk. The selected waveforms were compressed in time by a factor of $\sqrt{ } 3$ to satisfy the similitude requirements and scaled to the Peak Ground Acceleration (PGA) level of 0.259 g, which corresponds to the PGA value of the site according to the code assuming a topographic category T1. Scale Factors (SFs) of the acceleration time histories ranged from 0.73 to 1.43 . The selected ground motions are listed in Table 4 and the scaled 5\% damped elastic spectra are illustrated in Figure 5. 


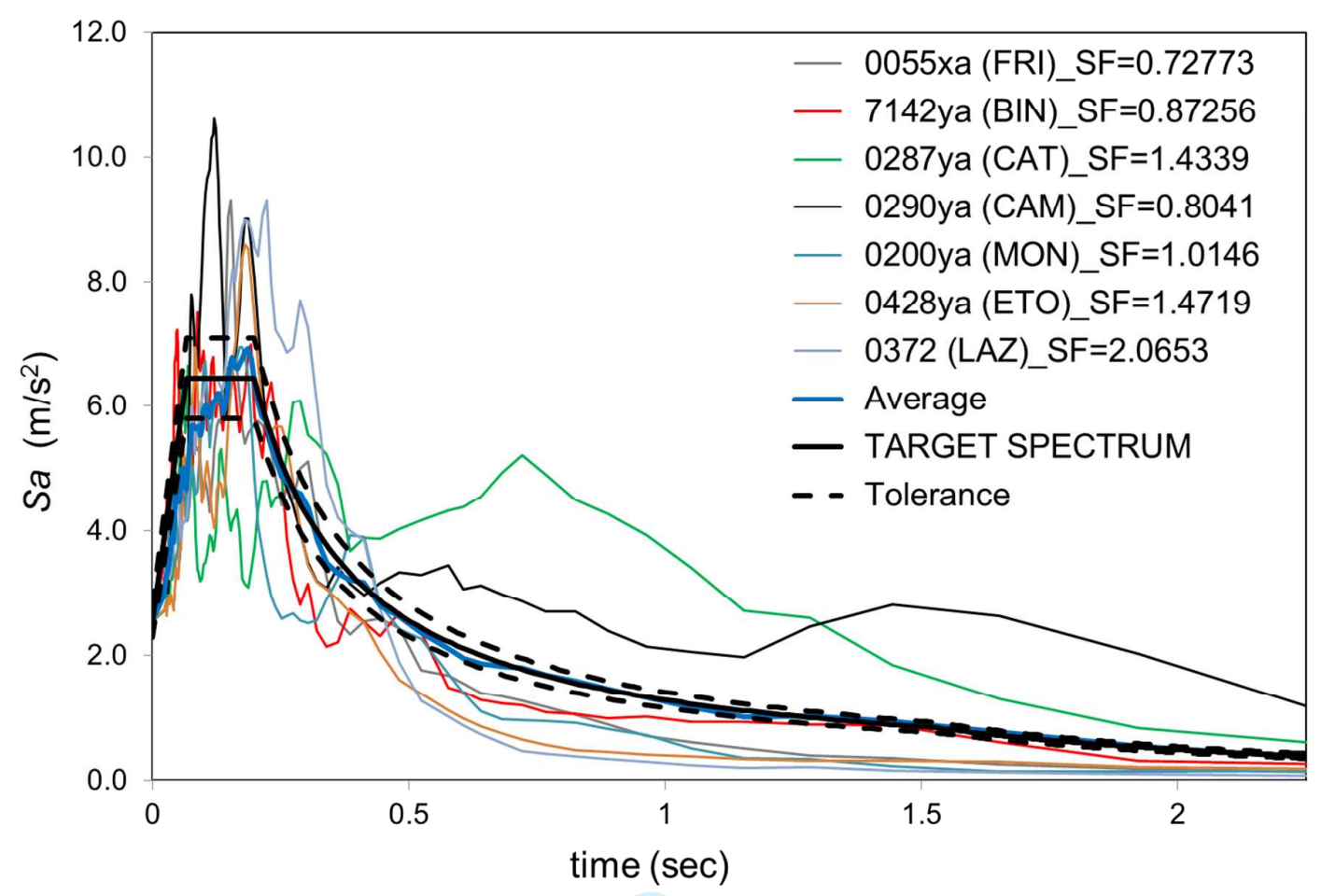

Figure 5. Scaled ground motion spectra and target spectrum according to Italian Building Code $\left(S_{T}=1 / 1 / \sqrt{ } 3 ; \mathrm{SF}_{\text {mean }}\right.$ $=1.1981)$ 
Table 4. Selected ground motions and details.

\begin{tabular}{|c|c|c|c|c|c|c|c|c|c|c|}
\hline \multirow[b]{2}{*}{ Record } & & \multirow[b]{2}{*}{ Waveform ID } & \multirow[b]{2}{*}{ Station ID } & \multirow[b]{2}{*}{ Date, $\mathrm{dd} / \mathrm{mm} / \mathrm{yy}$} & \multirow[b]{2}{*}{ SF } & \multirow[b]{2}{*}{ Mw } & \multirow[b]{2}{*}{$R, \mathrm{~km}$} & \multicolumn{3}{|c|}{ scaled earthquake } \\
\hline & & & & & & & & PGA, $\mathrm{m} / \mathrm{s}^{2}$ & $\mathrm{PGV}, \mathrm{cm} / \mathrm{s}$ & PGD, $\mathrm{cm}$ \\
\hline Bingol & $(\mathrm{BIN})$ & 7142 ya & ST539 & $01 / 05 / 2003$ & 0.87 & 6.3 & 14 & 2.55 & 18.29 & 3.25 \\
\hline Friuli & (FRI) & $55 x a$ & ST20 & 06/05/1976 & 0.72 & 6.5 & 23 & 2.55 & 15.25 & 9.29 \\
\hline Montenegro & $(\mathrm{MON})$ & $200 y a$ & ST68 & $15 / 04 / 1979$ & 1.01 & 6.9 & 65 & 2.55 & 12.87 & 9.60 \\
\hline Etolia & (ETO) & 428ya & ST169 & $18 / 05 / 1988$ & 1.47 & 5.3 & 23 & 2.55 & 12.46 & 6.06 \\
\hline Lazio Abruzzo & (LAZ) & 372 yа & ST274 & 07/05/1984 & 2.06 & 5.9 & 68 & 2.55 & 15.02 & 6.80 \\
\hline Campano Lucano & $(\mathrm{CAM})$ & $290 y a$ & ST96 & 23/11/1980 & 0.80 & 6.9 & 32 & 2.55 & 44.10 & 16.20 \\
\hline Campano Lucano & $(\mathrm{CAT})$ & $287 \mathrm{ya}$ & ST93 & $23 / 11 / 1980$ & 1.43 & 6.9 & 23 & 2.55 & 43.90 & 14.00 \\
\hline mean & & & & & 1.19 & 6.4 & 35 & & & \\
\hline
\end{tabular}




\section{Shake table tests}

Two protocols were performed within the present investigation.

The first, or "single event", experimental protocol aimed at verifying the restoring capability of the isolation system comprised of low dissipation Curved Surface Sliders. The mock-up was isolated with four CSS bearings with L-TF pads and subjected to the series of the seven one-directional ground motion records listed in Table 5. Each record was run once. The isolators were centred at the beginning of the series and if necessary in case of substantial residual displacement. Because of the huge displacements (restrained by the safety blocks) produced by the 290ya waveform, the record was scaled to $50 \%$ of the reference PGA.

The second, or "motion sequence", experimental protocol aimed at investigating the accrual of displacements due to an initial offset. The protocol was performed on the three configurations of the isolation system assessed in the characterization tests (Table 3). The detail of the ground motion sequences is given in Table 5: the BIN (7142ya), the CAT (287ya), and the FRI (55xa) records were run three to five times each; the CAM (290ya) record was run five times only for the configuration with high friction NL-CM pads. The isolators were in undeformed configuration before the beginning of the sequences. The possible residual displacement at the end of each run of ground motion was not removed, but remained as the initial offset at the beginning of the next run. Assuming that the input energy was identical in each run of the same ground motion record, the dependence of the maximum displacement on the initial offset was appraised. 
Table 5. Test sequence of ground motions: order of events and number of runs

\begin{tabular}{cccc}
\hline $\begin{array}{c}\text { Ground motion } \\
\text { record }\end{array}$ & L-TF & L-CM & NL-CM \\
\hline BIN & 3 & 5 & 5 \\
CAT & 3 & 5 & 5 \\
FRI & 3 & 3 & 5 \\
CAM & $=$ & $=$ & 5 \\
\hline
\end{tabular}

The response of the isolated structure was assessed in terms of relative displacements respect to the shake table and of absolute accelerations. The relative displacements were obtained by subtracting the shake table motion from the absolute displacements measured by the laser sensors fixed to the external frame. At each floor, the displacement of the structure in the direction of motion was calculated by averaging the displacements measured at two corners, see Figure 3(b). Absolute accelerations were directly measured by the accelerometers.

As an example, Figure 6 illustrates the time histories of the ground acceleration measured by the accelerometer on the shake table and the mock-up displacement at the base level during a run of the CAT (287ya) ground motion record for the three CSS configurations. The effect of the coefficient of friction in limiting the structure displacements through the mechanism of energy dissipation but at the same time reducing the re-centring capability is evident: the residual displacement is insignificant with low friction sliders (L-TF pads), small with medium friction sliders (L-CM pads) whereas it is a significant amount of the maximum displacement with high friction sliders (NL-CM pads). 

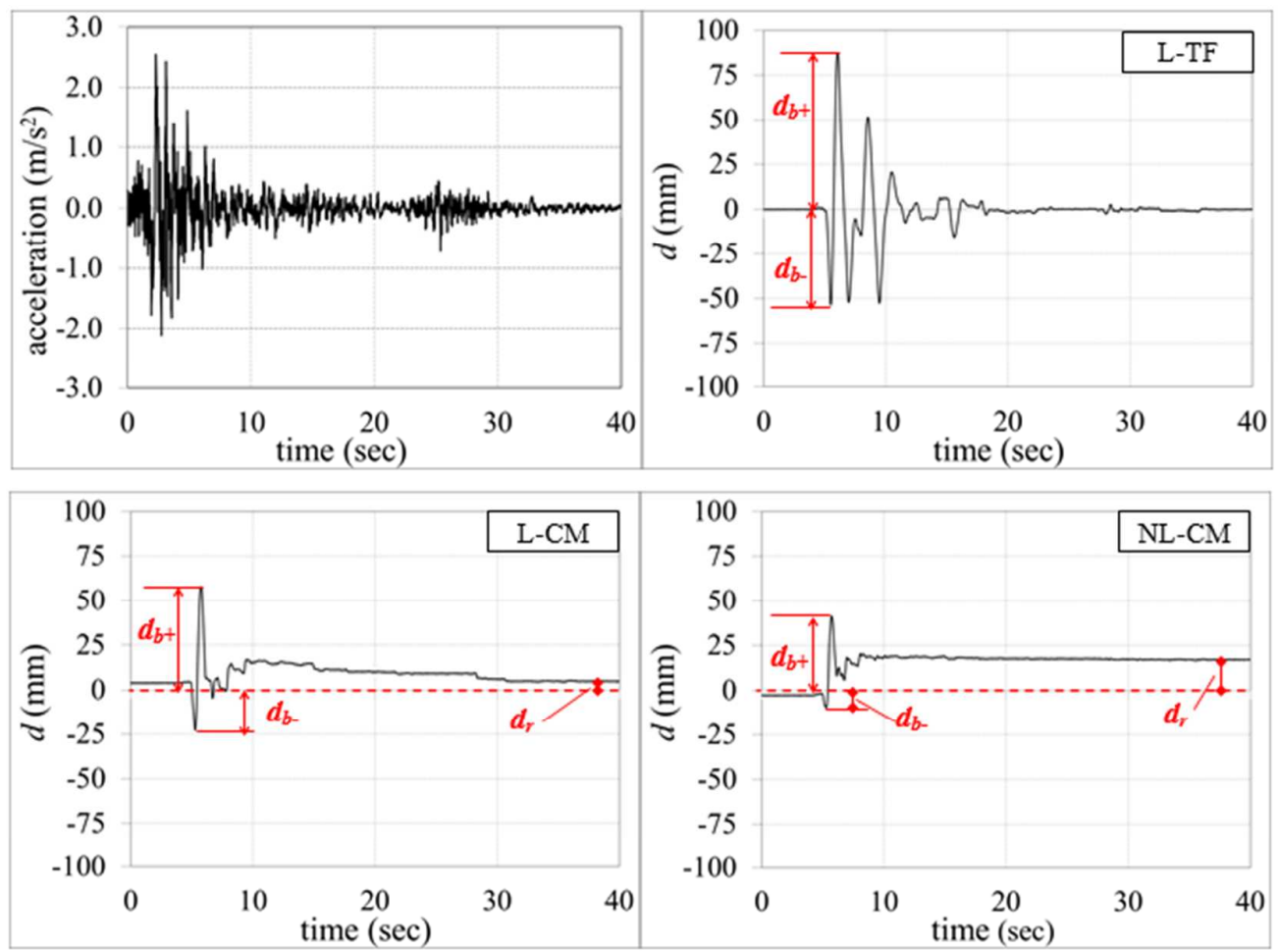

Figure 6. Time histories of shake table acceleration and structure base displacement during a run of CAT (287ya) ground motion record 
Results

The results are analysed accounting for the following quantities: the peak displacements $d_{b+}, d_{b-}$ of the structure at the isolation base level in either direction of motion, the maximum displacement $d_{\max }$ $=\max \left(d_{b^{+}},\left|d_{b_{-}}\right|\right)$, the residual displacement $d_{r}$, and the peak absolute acceleration $\left(a_{\max }\right)$ at the top floor of the structure. $d_{b^{+}}, d_{b^{+}}$and $d_{r}$ are in Figure 6.

The results of the "single event" protocol are shown in Figure 7. Assuming the largest maximum displacement over the seven ground motions $\left(d_{\max }=88.7 \mathrm{~mm}\right.$, CAT record $)$ as the design displacement $d_{c d}$ of the isolation system, for the CSS configuration with L-TF pads the ratio $d_{c d} / d_{0}$ results equal to $7.38\left(\mu_{\text {slow }}=0.008\right.$ as given in Table 2$)$. The residual displacement after a single earthquake becomes smaller with the increasing of the maximum displacement $d_{\text {max }}$, and the largest residual over the seven independent ground motions $(3.5 \mathrm{~mm}$, CAM record) is less than $4 \%$ of the assumed $d_{c d}$.

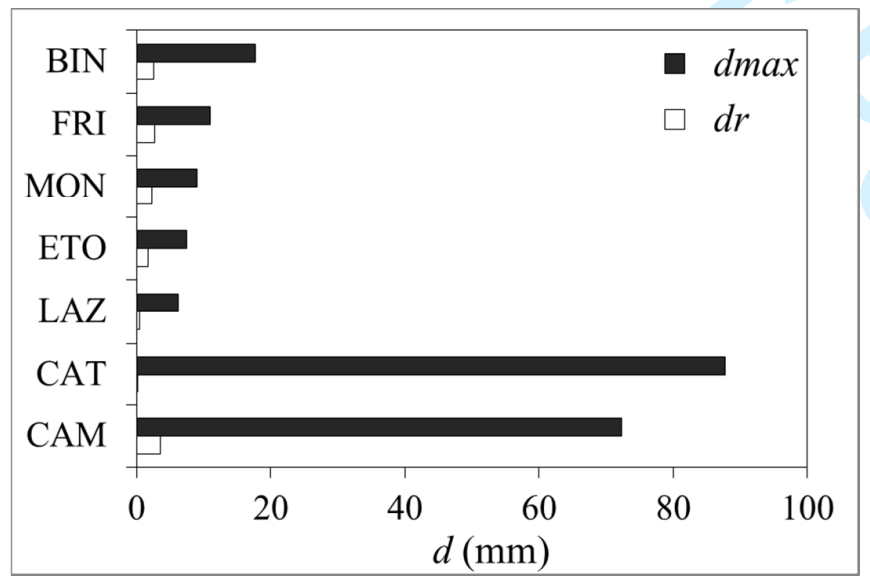

Figure 7. Maximum and residual absolute displacements of the CSS isolation system with low friction (L-TF) pads over seven spectrum-compliant ground motions. 
For the analysis of the results of the "motion sequence" protocol, the design displacement $d_{c d}$ of the isolation system was assumed as the displacement recorded at the first run of the 287ya (CAT) ground motion. As shown in Table 6, the code requirement $d_{c d} / d_{0}>0.5$ [17] was always fulfilled (relevant level of $\mu_{\text {slow }}$ as given in Table 2).

Table 6. Evaluation of the re-centring capability of the CSS isolation system according to the Eurocode 8 depending on the friction coefficient of the sliders

\begin{tabular}{cccc}
\hline sliding pad & $d_{c d}, \mathrm{~mm}$ & $d_{0}, \mathrm{~mm}$ & $d_{c d} / d_{0}$ \\
\hline L-TF & 87.7 & 11.9 & 7.38 \\
L-CM & 61.3 & 32.7 & 1.88 \\
NL-CM & 41.2 & 48.0 & 0.86 \\
\hline
\end{tabular}

Peak and residual displacement time-histories of the test sequences are illustrated in Figure 8, which reports, for every run of ground motion, the peak displacements $d_{b+}, d_{b \text {-, }}$, the initial offset $d_{i}$ and the residual displacement $d_{r}$ (the initial offset at the beginning of the next run).

The accrual of residual displacements is not significant for the CSS system with L-TF pads (Figure 8(a)).

Enough re-centring capability is shown also by the Curved Surface Sliders with L-CM pads (Figure 8(b)). The small offset at the beginning of the sequence of CAT ground motion remains unchanged during the whole sequence. The offset at the beginning of the FRI sequence is progressively recovered during three runs of the ground motion, with a corresponding decrease of the maximum displacement in each run.

On the contrary, insufficient re-centring capability is demonstrated by the CSS system with NLCM pads under the CAM and CAT sequences (only the displacement time-histories during the first sequence are reported due to similar trends), as reflected by the displacement growth (Figure 8(c)). 
1

2

3

4

5

6

7

8

9

10

11

12

13

14

15

16

17

18

19

20

21

22

23

24

25

26

27

28

29

30

31

32

33

34

35

36

37

38

39

40

41

42

43

44

45

46

47

48

49

50

51

52

53

54

55

56

57

58

59

60

At the end of the first run of ground motion a residual deformation occurs, which further increases at the end of the second and of the third run. The same behaviour is reflected by the maximum displacement of the system; however, the displacement build-up seems to end after the third run (Figure 9(a)). On the contrary, at the end of each run of the FRI ground motion sequence the system progressively returns towards its origin (Figure 9(b)) involving a decrease in the displacement amplitude; this behaviour is justified by Equation (10) as $\Delta d_{i}$ and $d_{\max }$ have opposite signs. 

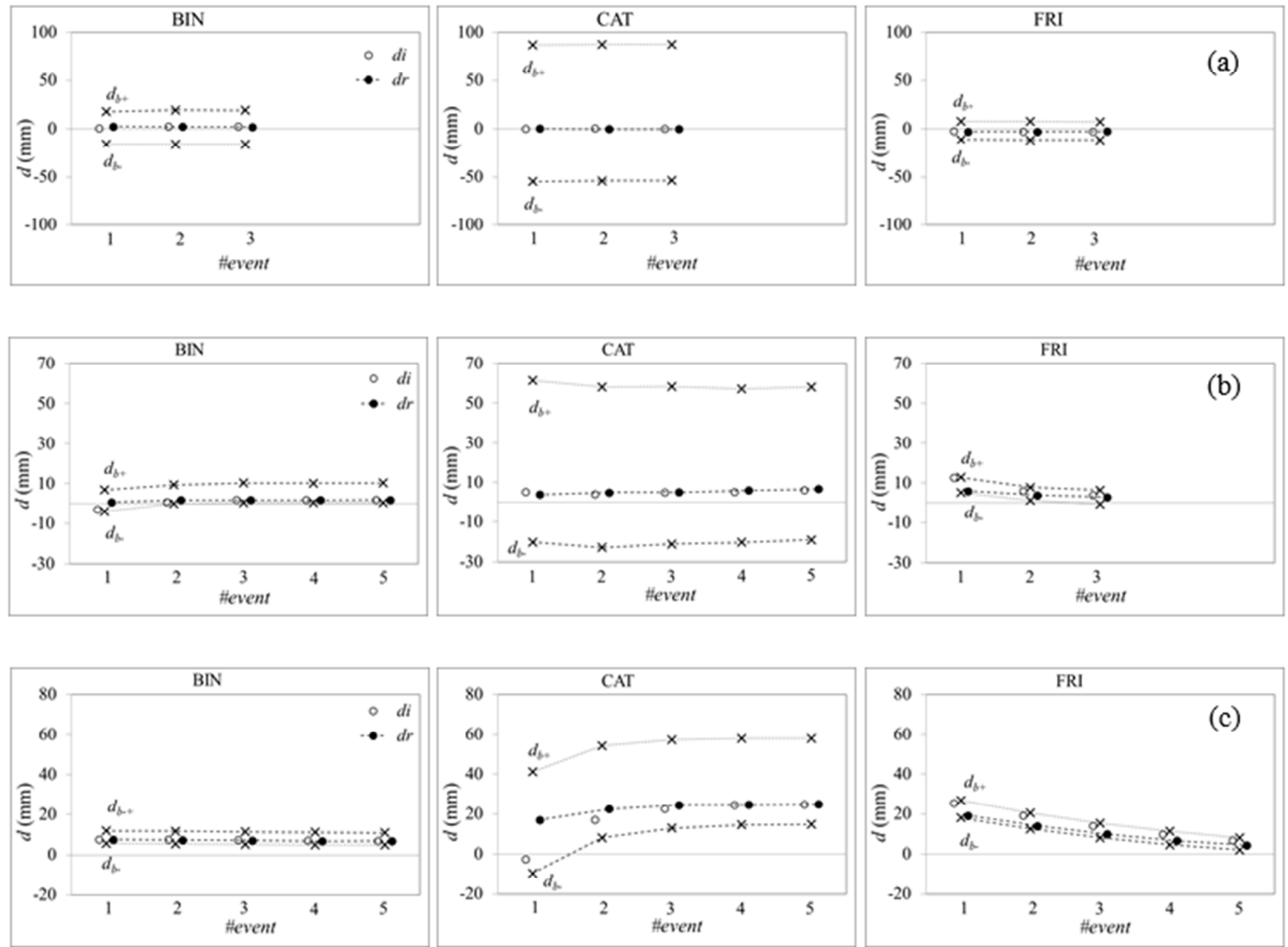

Figure 8. Peak displacement in either direction of motion $\left(d_{b+}, d_{b-}\right)$ and residual displacement $\left(d_{r}\right)$ depending on the initial offset $\left(d_{i}\right)$ during sequences of identical ground motion records: CSS system with L-TF pads (a), L-CM pads (b), and NL-CM pads (c).

Finally, Figure 10 shows the acceleration amplification factor, i.e. the ratio of the peak acceleration at the top floor of the structure to the peak ground acceleration. The largest accelerations occur for the isolators with high friction NL-CM pads, but remain nearly constant throughout each ground motion sequence, notwithstanding either the growth or decrease of permanent deformation. At least for the considered ground motion time histories, the acceleration amplification is not sensitive to the initial eccentricity of the isolation system, nor to the displacement accrual, and this result seems to be independent on the re-centring capability of the isolators. 

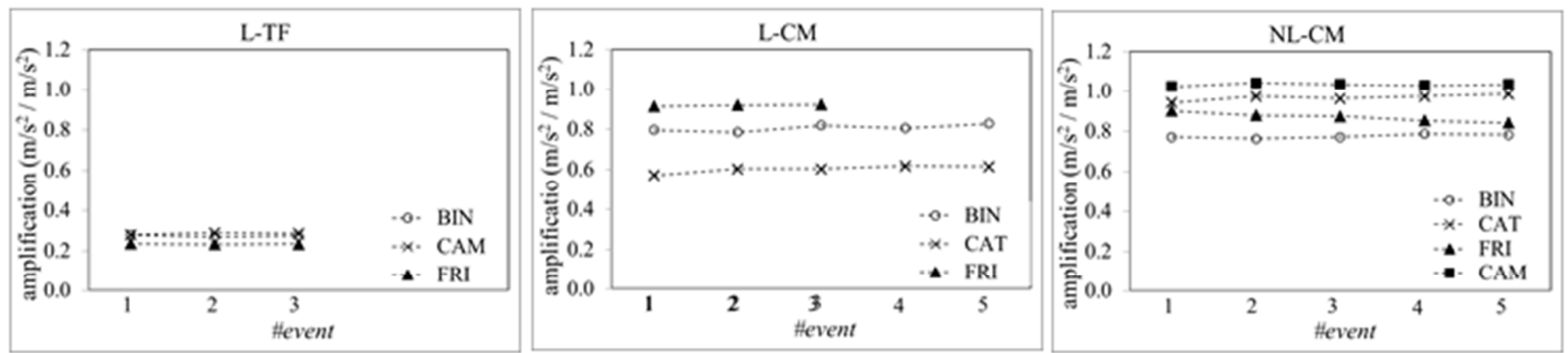

Figure 10. Acceleration amplification at the top floor of the structure 


\section{DISCUSSION}

Studies on the re-centring capability of bilinear hysteretic systems had been performed in the past [3], under the implicit assumption that the properties of the system do not change during the seismic event. Similar analyses were the basis for the re-centring criteria formulated in current codes [17]. It is therefore an argument whether or not the codes' provisions are valid for the Curved Surface Slider, because of the variability of the coefficient of friction during operation depending on the axial load, the velocity and the temperature.

To comply with the available testing facility, the present study was conducted on a one-third scaled structural mock-up of about $8000 \mathrm{~kg}$ mass, under the application of uni-directional ground motion time histories. The isolating devices were scaled to respect the geometric similitude requirements, but due to the strong nonlinearities introduced by friction, it is questionable how much the response of the scaled prototypes reflects the behaviour of real-scale isolators [38]. The unidirectional excitation represents another important simplification since, during the earthquake, isolation devices are in general subjected to the effect of general three-dimensional acceleration time histories. Biaxial tests performed on sliding isolators with curved surfaces $[39,40]$ proved the nonnegligible effects of the two horizontal components of motion that increase the heating of the sliding surfaces, leading to lower friction and consequently damping. These latter effects are expected to foster the re-centring of the devices, because result in increased displacement amplitude $d_{\max }$ and smaller value of $d_{0}$. Intuitively, also the vertical excitation of earthquakes should affect the horizontal response of a structure isolated with curved surface sliders since the horizontal force is proportional to the instantaneous vertical force. However, the experimental evidence seems to confirm that the coupling phenomenon between horizontal and vertical excitation can influence the horizontal response of the isolated structure in terms of shear forces, whereas it has little influence on the horizontal displacement [41-44]. 
The investigation presented in the study, though affected by the above limitations, has is the merit of being, to the knowledge of the authors, one of the first experimental studies addressing the effect of the coefficient of friction on the restoring capability of Curved Surface Sliders and the possible displacement accrual under a sequence of shakes.

The shake table tests point to the fact that also for the CSS system the re-centring capability improves as the parameter $d_{c d} / d_{0}$ increases, but the provision $\delta=0.5$ recommended by the code [17] seems to be not conservative for isolators with high friction materials, as the friction force can effectively contrast the re-centring action:

(a) The CSS isolation system with either low or medium friction coefficient demonstrated inherent re-centring capability, with small residual displacements in comparison to the design level $\left(d_{r} / d_{c d}\right.$ less than 0.04 and less than 0.10 respectively), and a not significant increase in the maximum deformation regardless of the initial eccentricity $\Delta d_{i}$; this result is in agreement with the recentring criterion of the Eurocode 8 based on the requirement $d_{c d} / d_{0} \geq 0.5$;

(b) The CSS isolation system with high friction, though meeting the code requirement $\left(d_{c d} / d_{0}=\right.$ 0.86), demonstrated an insufficient restoring behaviour: the permanent displacement can be an important part of the design displacement, possibly affecting the entity of the maximum displacement in a future event: e.g. at the end of the CAT ground motion sequence the permanent displacement was more than $50 \%$ of the maximum displacement $\left(d_{r}=0.56 d_{c d}\right)$, and the displacement amplitude increase of about $40 \%$ respect to the value at the first shacking .

Though the number of CSS configurations (only one effective radius $R_{b}$ was investigated) and ground motion time histories considered in the study is very limited and a generalization of the results to different situations cannot be made, some considerations can be drawn. First, the build-up of residual displacements always ended even in the unfavourable event in which the residual displacement of each earthquake was additive to the accumulated residual displacement of the previous earthquakes. Second, the peak displacement in either direction of motion, and hence the 
maximum displacement $d_{\max }$ during the earthquake, is influenced by the initial eccentricity $d_{i}$. An offset from the origin $\Delta d_{i}$ produces variations $\Delta d_{b+}$ and $\Delta d_{b-}$ that have the same sign of $\Delta d_{i}$ so that the displacement time history of the isolation system is shifted in the same direction of the eccentricity, and the larger $\Delta d_{i}$, the larger $\Delta d_{\max }$; this is illustrated in Figure 9 and is in agreement with Equation (10). Nevertheless, in some situations the ground motion may have a restoring effect and tends to re-centre the isolation system (e.g. Figure 9(b)). In section 2 it was explained that this is likely to occur especially for low intensity earthquakes owing to the contribution of the restoring force $K_{\mathrm{P}} \cdot \Delta d_{i}$, always directed towards the origin, to the resisting force at breakaway. This confirms that the re-centring capability does not depend on the mechanical characteristics of the isolation system alone, but also on the details of the seismic waveform.

According to the codes, the displacement demand is calculated based on the single occurrence of the design earthquake, and without consideration of any initial offset. For Curved Surface Sliders with good re-centring capability the displacement demand does not change significantly even in the event of a seismic sequence comprising pre-shocks or aftershocks. But for systems with low selfcentring capability large residual displacement can occur under this circumstance and possibly jeopardize the displacement capacity of the device if the likelihood of displacement accrual was not adequately accounted for during their design.

It is worth noting that some practical issues have to be considered which require a more prudential estimate of the re-centring capability. First, the coefficient of friction tends to increase with wear and contamination of the sliding surfaces, like e.g. in case of inadequate protection from dust, and consequently the re-centring capability must reasonably be expected to decrease during the lifetime of CSSs. Also the "stick slip" phenomenon can lead to a dramatic decrease in the restoring behaviour of the friction devices. Eventually, the presence of an internal articulation in the curved surface slider that allows independent movement of the sliding surfaces from each other can make unpredictable the restoring behaviour of the system. The tests presented in the study were performed on prototypes 
with only two pairs of curved sliding surfaces and a rigid slider, so due to kinematic constraints the two surfaces slide at the same time. Sliders with more than two pairs of sliding surfaces and/or an internal articulation can exhibit delays in either the beginning or stop of sliding of one the sliding surfaces, due e.g. to differences in the coefficient of friction at either surface. It is therefore proposed that a more conservative figure for the criterion $d_{c d} / d_{0}$ should be adopted for Curved Surface Slider isolators.

Though beyond the scope of the paper, it has to be remarked that the final evaluation of the recentring capability of current Curved Surface Sliders shall be performed in shake table tests on a building at the real scale, with real scale isolators and under the application of three dimensional excitations (along the two horizontal and the vertical directions) and possibly full scale real ground motion records. To the knowledge of the authors, the largest shake table is available at the Hyogo Earthquake Engineering Research Centre, otherwise known as E-Defense, of the National Research Institute of Earth Science and Disaster Prevention $[45,46]$. The shake table has a payload of $12 \mathrm{MN}$ and was used to perform tests on a full-scale $2 \times 2$ bay, 5-story moment frame building with dimensions $10 \times 12 \times 16 \mathrm{~mm}$ (width $\times$ length $\times$ height) isolated with triple friction pendulum bearings [41]. 


\section{Conclusions}

The re-centring capability of three isolation systems comprised of Curved Surface Sliders with same restoring stiffness but differing for the coefficient of friction was assessed in shake table tests on a scaled model of a building frame. The results of the tests can be summarized in the next points:

(1) the parameter $d_{c d} / d_{0}$ seems to govern the re-centring capability of the CSS system in accordance with the provisions stipulated in the Eurocode 8 [17]; however the criterion $d_{c d} / d_{0} \geq 0.5$ appears to be not conservative, especially for high friction isolators;

(2) since $d_{c d}$ includes the effects of seismic excitation, the re-centring capability of the Curved Surface Slider depends on the ground motion characteristics, resulting generally better for earthquakes promoting larger maximum displacements;

(3) an initial offset of the isolation system affects the maximum displacement occurring during the earthquake, but the effect is lower for systems with good re-centring capability; the quantities $\Delta d_{i}$ (initial eccentricity or offset of the system) and $\Delta d_{\max }$ (variation of maximum seismic displacement consequent to the offset) have the same sign and are related through the parameter $d_{c d} / d_{0}$

(4) depending on the characteristics of the waveform, the ground motion may either produce an accrual of displacement, or have a restoring effect on a displaced system, reducing the eccentricity at the end of the shaking;

(5) a conservative estimation of the displacement capacity of isolation systems with high friction is highly recommended, since when the design displacement is close to the displacement capacity of the isolators, a not negligible residual displacement may jeopardize the displacement capacity of the isolation system considering the occurrence of possible aftershocks and future earthquakes. Though not directly investigated, attention was also addressed to the fact that the wear and contamination of the sliding surfaces, the use of sliding materials with "stick-slip" behaviour, and the possible delays at the beginning of motion between the sliding surfaces, which are not predictable at 
the design stage, can possibly adversely affect the restoring capability of the Curved Surface Slider, requiring a more conservative re-centring criterion than recommended in the code.

Due to the small number of waveforms considered in the study, the influence of the ground motion characteristics was not investigated in detail, and the conclusions cannot be generalized. Nevertheless the study is of some value in that it is among the first experimental works addressing the influence of the coefficient of friction and the possible effect of a permanent deformation on the displacement accrual, and provides some pieces of factual information on the re-centring behaviour of curved surface sliding isolation systems which can be the basis for the development of more refined theoretical models.

\section{Conflict of Interests}

The authors declare that there is no conflict of interests regarding the publication of this paper.

\section{Acknowledgements}

The authors acknowledge the support provided by the Italian Department of Civil Protection (DPC) in the frame of the national Research Project DPC - ReLUIS (National Network of Laboratories of Seismic Engineering) 2014-2018, Line 6 - Isolation and Dissipation. 


\section{REFERENCES}

[1] Medeot R. Re-centring capability evaluation of seismic isolation systems based on energy concepts. Proceedings of the 13th World Conference on Earthquake Engineering 2004, paper 3106, Vancouver, Canada.

[2] Berton S, Infanti S, Castellano MG, Hikosaka H. Self-centring capacity of seismic isolation systems. Structural Control and Health Monitoring 2006; 4(6), pp. 895-914, DOI: 10.1002/stc. 188.

[3] Katsaras CP, Panagiotakos TB, Kolias B. Restoring capability of bilinear hysteretic seismic isolation systems. Earthquake Engineering and Structural Dynamics 2008; 37(4), pp. 557-575, DOI: 10.1002/eqe.772.

[4] ASCE/SEI 7 (revision for 2016) Minimum design loads for buildings and other structures, American Society of Civil Engineers, 1801 Alexander Bell Drive Reston, VA 20191.q

[5] Decanini L, Gavarini C, Mollaioli F. Some remarks on the Umbria-Marche earthquakes of 1997. European Earthquake Engineering 2000; 3, pp. 18-48.

[6] Huang Y, Wu JP, Zhang TZ, Zhang DN. Relocation of the M 8.0 Wenchuan earthquake and its aftershock sequence. Science in China (Series D) 2008; 51(12), pp. 1703-1711, DOI: 10.1007/s11430-008-0135-z.

[7] Di Sarno L, Elnashai AS, Manfredi G. Assessment of RC columns subjected to horizontal and vertical ground motions recorded during the 2009 L'Aquila (Italy) earthquake. Engineering Structures 2011; 33(5), pp. 15141535, DOI:10.1016/j.engstruct.2011.01.023.

[8] Di Sarno L, Yenidogan C, Erdik M. Field evidence and numerical investigation of the Mw $=7.1$ October 23 Van, Tabanli and the Mw > 5.7 November Earthquakes of 2011. Bulletin of Earthquake Engineering, 2013; 11(1), pp. 313-346, DOI: 10.1007/s10518-012-9417-0.

[9] Motosaka M, Mitsuji K. Building damage during the 2011 off the Pacific coast of Tohoku Earthquake. Soils and Foundations 2012; 52(5), pp. 929-944, DOI:10.1016/j.sandf.2012.11.012.

[10] Carydis P, Castiglioni C, Lekkas E, Kostaki I, Lebesis N, Drei A. The Emilia Romagna, May 2012 earthquake sequence. The influence of the vertical earthquake component and related geoscientific and engineering aspects. Ingegneria Sismica - International Journal of Earthquake Engineering 2012; XXIX, pp. 31-58.

[11] Riddell R, Newmark NM. Statistical analysis of the response of nonlinear systems subjected to earthquakes. Research Report, University of Illinois at Urbana, 1979.

[12] Mahin SA, Bertero VV. An evaluation of inelastic seismic response spectra. Journal of Structures 1981; 107(9), pp. $1777-1795$.

[13] Kawashima K, MacRae GA, Hoshikuma J, Kazuhiro N. Residual displacement response spectrum. Journal of Structural Engineering 1998; 124(5), pp. 523-530, DOI: 10.1061/(ASCE)0733-9445(1998)124:5(523). 
[14] MacRae GA, Kawashima K. Post-earthquake residual displacements of bilinear oscillators. Earthquake Engineering and Structural Dynamics 1997; 26(7), pp. 701-716, DOI: 10.1002/(SICI)10969845(199707)26:7<701::AID-EQE671>3.0.CO;2-I.

[15] Tsopelas P, Constantinou MC. NCEER - TAISEI corporation research program on sliding seismic isolation systems for bridges - experimental and analytical study of a system consisting of sliding bearings and fluid restoring force/damping devices. Technical Report NCEER-94-0014, National Center for Earthquake Engineering Research, 1994.

[16] Naeim F, Kelly JM. Design of seismic isolated structures. Wiley, 1999.

[17] CEN Eurocode 8: design of structures for earthquake resistance-Part 2: Bridges, EN1998-2:2005+A1:2011. European Committee for Standardization, Bruxelles, Belgium, 2005.

[18] CEN EN 15129: Anti-Seismic Devices. European Committee for Standardization, Bruxelles, Belgium, 2009.

[19] Zayas VA, Low SS, Mahin SA. The FPS earthquake protection system: experimental report. Report No. UCB/EERC-87/01, Earthquake Engineering Research Center, University of California at Berkeley, 1987.

[20] Zayas VA, Low SS, Mahin SA. A simple pendulum technique for achieving seismic isolation. Earthquake Spectra, 1990; 6(2), pp. 317-333, DOI: $10.1193 / 1.1585573$.

[21] Mokha AS, Constantinou MC, Reinhhorn AM. Experimental study of friction-pendulum isolation system. Journal of Structural Engineering 1991; 117(4), pp. 1201-1217, DOI: 10.1061/(ASCE)0733-9445(1991)117:4(1201).

[22] Constantinou MC, Whittaker AS, Kalpakidis Y, Fenz DM, Warn GP. Performance of seismic isolation hardware under service and seismic loading. Technical Report MCEER-07-0012, Multidisciplinary Center for Earthquake Engineering Research, 2007.

[23] Constantinou MC, Mokha A, Reinhorn AM. Teflon bearings in base isolation. II: modeling. Journal of Structural Engineering 1990; 116(2), pp. 455-474, DOI: 10.1061/(ASCE)0733-9445(1990)116:2(455)

[24] Dolce M, Cardone D, Croatto F. Frictional behaviour of steel-PTFE interfaces for seismic isolation. Bulletin of Earthquake Engineering 2005; 3(1), 75-99, DOI: 10.1007/s10518-005-0187-9.

[25] Quaglini V, Dubini P, Poggi C. Experimental assessment of sliding materials for seismic isolation systems. Bulletin of Earthquake Engineering 2012; 10(2), pp. 717-740, DOI: 10.1007/s10518-011-9308-9.

[26] Quaglini V, Bocciarelli M, Gandelli E, Dubini P. Numerical Assessment of Frictional Heating in Sliding Bearings for Seismic Isolation. Journal of Earthquake Engineering, 2014; 18(8), pp. 1198-1216, DOI: 10.1080/13632469.2014.924890. 
[27] Lomiento G, Bonessio N, Benzoni G. Friction Model for Sliding Bearings under Seismic Excitation. Journal of Earthquake Engineering 2013; 17(8), pp. 1162-1191, DOI:10.1080/13632469.2013.814611.

[28] Cardone D, Gesualdi G, Brancato P. Restoring capability of friction pendulum seismic isolation systems. Bulletin of Earthquake Engineering 2015; 13(8), pp 2449-2480, DOI: 10.1007/s10518-014-9719-5.

[29] Sarlis AA, Constantinou MC, Reinhorn AM. Shake table testing of Triple Friction Pendulum Isolators under extreme conditions. Technical Report NCEER-13-0011, National Center for Earthquake Engineering Research, 2013.

[30] Al-Hussaini TM, Zayas VA, Constantinou MC. Seismic isolation of a multi-story frame structure using spherical sliding isolation systems. Technical Report No. NCEER-94-0007, National Center for Earthquake Engineering Research, 1994.

[31] Medeot R. Lesson Learned from Shake Table Tests on Flat and Curved Surface Sliders. Proceedings of the Seventh World Congress on Joints, Bearings and Seismic Systems for Concrete Structures, 2011, Las Vegas, USA.

[32] Ismail M, Rodellar J, Pozo F. An isolation device for near-fault ground motions. Structural Control and Health Monitoring 2014; 21(3), pp. 249-268, DOI: 10.1002/stc. 1549.

[33] Calabrese A. Spizzuoco MC, Serino G, Della Corte G, Maddaloni G. Shaking table investigation of a novel, lowcost, base isolation technology using recycled rubber. Structural Control and Health Monitoring, 2015; 22(1), pp. 107-122, DOI: $10.1002 /$ stc. 1663 .

[34] Fenz DM, Constantinou MC. Behaviour of the double concave Friction Pendulum bearing. Earthquake Engineering and Structural Dynamics 2006; 35(11), pp. 1403-1424, DOI: 10.1002/eqe.589.

[35] NTC, 2008. Approvazione delle nuove norme tecniche per le costruzioni, Gazzetta Ufficiale della Repubblica Italiana n. 29 del 4 febbraio 2008 - Suppl. Ordinario n. 30, (in Italian).

[36] Ambraseys N, Smit P, Sigbjornsson R, Suhadolc P, Margaris B. http://www.isesd.cv.ic.ac.uk/ESD/ Internet-Site for European Strong-Motion Data, European Commission, Research-Directorate General, Environment and Climate Programme, 2002.

[37] Iervolino I, Galasso C, Cosenza E. REXEL: computer aided record selection for code-based seismic structural analysis. Bulletin of Earthquake Engineering 2010; 8(2), pp. 339-362, DOI: 10.1007/s10518-009-9146-1.

[38] Bozzo S, Harbat AH. Nonlinear response of structures with sliding base isolation. Structural Control and Health Monitoring 1995; 2 (2), pp. 59-77, DOI: 10.1002/stc.4300020203. 
[39] Fagà E, Ceresa P, Nascimbene R, Moratti M, Pavese A. Modelling curved surface sliding bearings with bilinear constitutive law: effects on the response of seismically isolated buildings. Materials and Structures $\mathbf{2 0 1 5}$ (published on line). DOI 10.1617/s11527-015-0642-2.

[40] Roussis PC, Constantinou MC. Experimental and analytical studies of structures seismically isolated with an uplift-restraining friction pendulum system. Earthquake Engineering \& Structural Dynamics 2006; 35, pp. 595611. DOI: $10.1002 /$ eqe.546.

[41] Ryan K, Dao N. Influence of vertical ground shaking on horizontal response of seismically isolated buildings with friction bearings. Journal of Structural Engineering 2016; 142(1), DOI: 10.1061/(ASCE)ST.1943-541X.0001352, 04015089.

[42] Mosqueda G, Whittaker AS, Fenves GL. Characterization and modeling of friction pendulum bearings subjected to multiple components of excitation. Journal of Structural Engineering 2004; 130(3), pp. 433-442, DOI: 10.1061/(ASCE)0733-9445(2004)130:3(433).

[43] Morgan TA, Mahin SA. The use of innovative base isolation systems to achieve complex seismic performance objectives. Technical Report No. PEER-2011/06, Pacific Earthquake Engineering Research Center, 2011,

[44] Fenz DM, Constantinou MC. Development, implementation and verification of dynamic analysis models for multi-spherical sliding bearings. Technical Report No, MCEER-08-0018, Multidisciplinary Center for Earthquake Engineering Research, NY, 2008.

[45] Ogawa N, Ohtani K, Katayama T, Shibata H, Construction of a three-dimensional, large-scale shaking table and development of core technology. Philosophical Transactions of the Royal Society A 2001; 359, pp. 1725-1751, DOI: DOI: 10.1098/rsta.2001.0871.

[46] Ohtani K, Ogawa N, Katayama T, Shibata H. Construction of E-Defense (3-D full-scale earthquake testing facility). Proceedings of the 13th World Conference on Earthquake Engineering 2004, paper 189, Vancouver, Canada. 


\title{
Experimental investigation of the re-centring capability of Curved Surface Sliders
}

\author{
Virginio Quaglini ${ }^{(*)}, \mathrm{PhD}$, Emanuele Gandelli, MSc, Paolo Dubini, PhD \\ Politecnico di Milano, Department of Architecture, Built Environment and Construction \\ Engineering. Piazza Leonardo da Vinci 32, 20133 Milano \\ ${ }^{(*)}$ corresponding author \\ Email: virginio.quaglini@polimi.it \\ Phone: +39 02.2399.4248
}




\section{ABSTRACT}

The re-centring capability is recognized as a fundamental function of the isolation system, since it is intended to prevent substantial permanent deformation at the end of the earthquake that may affect the serviceability of the structure and eventually limit the capability of the isolators to withstand aftershocks and future earthquakes. In this study the re-centring behaviour of isolation systems comprised of sliding bearings with curved surfaces is investigated in shake table tests carried out on a one-story steel frame with rectangular plan, scaled at one third-length scale and isolated with four bearings. The coefficient of friction of the bearings is varied by changing the material or lubrication condition of the pads, providing different equivalent damping ratios to the isolation system. The response of the base isolated structure to selected natural ground motion waveforms is assessed in terms of the residual displacement after a single event and the accrual of displacements during a sequence of quakes, and considerations on the influence of the coefficient of friction on the recentring behaviour, as well as on the effect of an initial displacement offset are drawn. The recentring provision of the current European design code is eventually checked against the experimental data.

Keywords: base isolation; curved surface sliders; re-centring capability; shake-table test; displacement accrual; residual displacement 


\section{INTRODUCTION}

Base isolation is a modern strategy for seismic protection based on the provision of the construction with a set of bearings that decouple the motion of the superstructure from the motion of the ground. The bearings are aimed at modifying the response of the superstructure by lengthening its fundamental period, which reduces forces but increases displacements, and by increasing damping, which reduces displacements and may reduce forces.

A fundamental function of the base isolation system is recognized to be the ability to return towards the origin at the end of ground motions or tremors, or self re-centring [1-3]. This function is associated with the prevention of large residual displacement after the earthquake, which may affect the serviceability of the structure and jeopardize the functionality of elements crossing the isolation plane (like fire protection and weather proofing elements, egress/entrance details, elevators, joints of primary piping systems etc.), and eventually limit the capability of the isolation system to withstand aftershocks and future earthquakes [4]. There is significant field evidence of seismic sequences characterized by frequent medium-strong intensity ground motions following a strong mainshock after short intervals of time [5-10]. Since it may not be possible to re-center the system before close aftershocks, ground motion sequences with such characteristics would entail an accrual of displacements, and the deformation capacity of the isolation system designed based on a single earthquake possibly become inadequate.

In the past, structural engineers did not pay much attention to the re-centring capability of seismic isolators, and the first studies focused on the residual displacements of generic low-ductility nonlinear SDOF systems [11-14]. This was probably a consequence of the fact that the first seismic isolators were conventional laminated rubber bearings that were endowed with an inherent selfcentring capability associated with the elastic restoring force developed in the rubber layers subjected to shear deformation [15-16]. However in the recent years, with the introduction in the market of new isolation devices with eharacterized by high energy dissipation capacity like lead 
2

rubber bearings, sliding isolators with steel hysteretic elements, friction bearings, etc., the problem of limiting residual displacements turned out of primary interest. Criteria for evaluating the re-centring capability of the isolation system have been provided in modern seismic codes. According to the European code, or Eurocode 8 [17], this capability is available in one horizontal direction when the system has small residual displacements in relation to its displacement capacity, and the requirement is considered satisfied when the condition is met:

$$
\frac{d_{c d}}{d_{0}} \geq \delta
$$

where $d_{c d}$ is the design displacement of the isolation system in the examined direction, $d_{0}$ is the "maximum static residual displacement" for which the isolation system can be in static equilibrium in the considered direction, i.e. the residual displacement under which the static equilibrium is reached at unloading from $d_{c d}$ under quasi-static conditions (Figure 1), and $\delta$ is a numeric coefficient, whose recommended value is 0.5 .

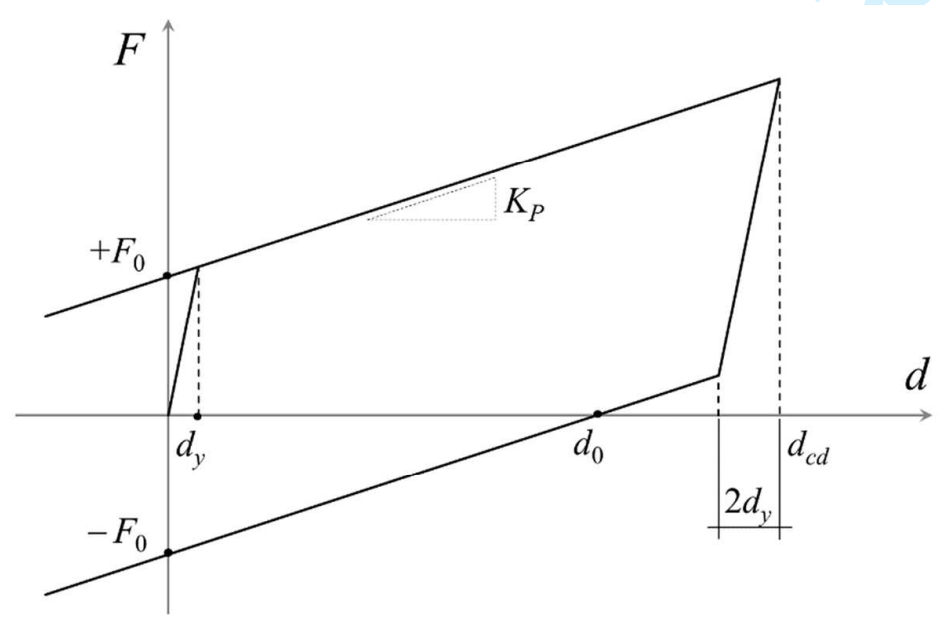

Figure 1. Equivalent bilinear hysteretic model of the isolation system for the evaluation of the re-centring capability

The validity of this criterion for isolation systems with the typical bilinear hysteretic behaviour shown in Figure 1 was assessed in a parametric study [3], analysing 150 different combinations of 
system parameters and 122 natural ground motions. Systems with $d_{c d} / d_{0} \geq 0.5$ demonstrated small residual displacements compared with the maximum displacement induced by the ground motion, and insignificant accumulation of residual displacements at the end of a sequence of earthquakes. Similarly, an experimental study on isolation systems for bridges comprised of flat sliding bearings, rubber devices and fluid dampers [15] showed that the a strong re-centring capability is provided when the ratio of the characteristic strength $F_{0}$ to the peak restoring force at the maximum displacement $d_{\max }$, i.e. $K_{P} d_{\max }$, is less than or equal to 3 , which corresponds to $d_{\max } / d_{0} \geq 0.3$ ( $F_{0}$ and $K_{P}$ defined as in Figure 1).

The European design code requires that systems that do not satisfy the re-centring provision of Equation (1), have anyway sufficient displacement capacity to accommodate, with adequate reliability, the accrual of residual displacements during the service life of the structure. To account for the possible displacement build-up ef residual displacements under a sequence of earthquake events occurring before the design earthquake,-considered to have a collective probability equal to the probability of the design earthquake. The the displacement demand must be increased by a factor

$$
\rho_{d}=1+1.35 \frac{1-\left(d_{y} / d_{c d}\right)^{0.6}}{1+80\left(d_{c d} / d_{0}\right)^{1.5}}
$$

where $d_{y}$ is the yield displacement (see Figure 1) of the equivalent bilinear system. For systems with $d_{c d} / d_{0}>0.5, \rho_{d}<1.05$ and the effect of the accumulation of residual displacements is insignificant.

A second re-centring criterion, based on energy concepts, is provided in the European standard on antiseismic devices EN 15129 [18]: the system has sufficient re-centring capability when the relation is met

$$
E_{S} \geq 0.25 E_{H}
$$

where $E_{S}$ is the reversibly stored elastic energy and $E_{H}$ is the energy dissipated in hysteretic deformation when the isolation system moves from its origin to the position of maximum 
displacement. It can be easily demonstrated that for bilinear hysteretic systems the provision of Equation (3) is identical to Equation (1) where $\delta=0.5$.

Among the most popular antiseismic hardware today there is the Curved Surface Slider (CSS), which was firstly introduced in North America in the second part of the '90s in the version called the Friction Pendulum System ${ }^{\circledR}$, or FPS $^{\circledR}$ [19-21], and whose wide use is in progress in Europe and worldwide after the expiry of patent rights in 2005. The Curved Surface Slider is based on the principle of accommodating the horizontal displacement of the structure by means of one of more pair(s) of curved sliding surfaces, which provide a restoring force due to the effect of the curvature and dissipate seismic energy through friction forces developed during sliding. Energy dissipation and self-centring capability are two antithetic functions, as friction forces can act away from the origin contrasting the effect of the restoring force [1], and a potential issue that has come recently under attention is the occurrence of large residual displacements when materials with high levels of friction are used in the sliding surfaces. This concern is further of particular importance when the sliding material exhibits the so-called "stick-slip" phenomenon, i.e. a continuous transition from the sliding phase to the sticking phase and vice versa occurring at slow velocities [22]: in the sticking phase the coefficient of friction rises from its dynamic (or kinetic) level to the static level hence increasing the resistance to the re-centring motion of the isolating system.

In the light of these considerations, it is therefore questionable whether the re-centring criterion of the Eurocode 8 is valid for Curved Surface Sliders. The criterion was indeed formulated under the assumption that the mechanical properties of the isolation system, lumped in the parameter $d_{0}$ in Equation (1), are constant. This is in general not true for the CSS, as the coefficient of friction is noted to be dependent on a number of factors among which there are the axial load, the velocity of sliding and the temperature [23-27]. Research addressing the re-centring capability of Curved Surface Sliders is very recent, and definite results have not yet been achieved. A parametric study [28]pointed that the code's provision seems to be not conservative and sliding isolators with curved 
surfaces experience negligible residual displacements only when $d_{c d} / d_{0}$ is larger than 2.5 . Also the experimental knowledge is little. Shake table tests on a 3-story seismically isolated structure equipped with triple friction pendulum isolators and simulating sequences of low-to-moderate and high amplitude seismic ground motions, concluded that depending on the sequence details, the isolation system may either accumulate or recover residual displacements [29].

The evaluation of the self-centring capability of the Curved Surface Slider is of primary importance since it can significantly affect the design of the isolation system, as well as of the isolated structure that must be designed to accommodate the residual displacements. The present study aims at giving an insight into the matter, providing the results of an experimental investigation conducted within the framework of the Task 6 "Isolation and Dissipation" of the Italian RELUIS (National Network of Laboratories of Seismic Engineering) Project 2010-2013. The two main goals are: (1) to evaluate the re-centring capability of curved surface sliders comparing devices with different coefficient of friction, and (2) to assess the influence of permanent displacements on the amplitude of the seismically induced motion and the possible accrual of displacements. 


\section{Theoretical considerations}

The force - displacement behaviour of the Curved Surface Slider is conventionally described [30] in accordance with the bilinear hysteretic model illustrated in Figure 1, where $F_{0}$ is the characteristic strength and $K_{P}$ is the restoring stiffness of the isolator. Both properties depend on the characteristics of the curved surfaces according to the relations $F_{0}=\mu_{d} \cdot N$ and $K_{P}=N / R_{b}$ where $N$ is the vertical load acting through the bearing, $\mu_{d}$ is the dynamic coefficient of friction, and $R_{b}$ is the effective radius of curvature [30]. For the Curved Surface Slider the yield displacement $d_{y}$ is usually negligible and can be assumed to be zero [17]. The horizontal reaction force $F$ of the isolator at a displacement $d$ is therefore given by:

$$
F=F_{0} \cdot \operatorname{sign}(\dot{d})+K_{P} \cdot d
$$

where $\operatorname{sign}(\dot{d})$ is the sign of the velocity vector.

The static equilibrium between the restoring force $K_{P} \cdot d$ that always acts towards the origin, and the friction force $\mu_{d} \cdot N$ that contrasts the motion is attained at the displacement $d_{0}$

$$
d_{0}=\frac{F_{0}}{K_{P}}=\mu_{d} \cdot R_{b}
$$

which represents the upper bound to the actual residual displacement $d_{r}$ at the end of the motion and is independent on the displacement capacity of the isolator. Assuming for simplicity that the motion of the bearing is symmetric with respect to the origin, with amplitude equal to $d_{c d}$, then the effective damping can be expressed as

$$
\xi_{b}=\frac{2}{\pi} \cdot \frac{\mu_{d} \cdot R_{b}}{d_{c d}}=\frac{2}{\pi} \cdot \frac{d_{0}}{d_{c d}}
$$

Equation (6) confirms that increasing the energy dissipation capacity of the bearing entails a corresponding decrease in the re-centring capability expressed by the ratio $d_{c d} / d_{0}$.

For the Curved Surface Slider the provisions (1) and (3) can be reformulated as [31] 


$$
\frac{d_{c d}}{\mu_{d} \cdot R_{b}} \geq 0.5
$$

The dependency of the coefficient of friction on the sliding velocity has a fundamental importance in the response of the isolation system during the earthquake ground motion. According to a widely recognized model [23], this dependency is expressed through the exponential law

$$
\mu_{d}=\mu_{\text {fast }}-\left(\mu_{\text {fast }}-\mu_{\text {slow }}\right) \cdot \exp (-\alpha \cdot V)
$$

where $\mu_{\text {slow }}$ and $\mu_{\text {fast }}$ are the levels of the friction coefficient at low and fast velocities, respectively, $V$ is the sliding velocity and $\alpha$ is a rate parameter, with dimensions of the inverse of velocity, which depends on axial load and air temperature [24]. The coefficient of friction increases more than linearly with increasing the velocity, and the value of $\mu_{\text {fast }}$ is important in determining the maximum displacement of the isolation system that is achieved during the strong motion stage of the earthquake when the system absorbs energy. On the contrary, a lower level of friction governs the response of the system during the coda stage of the earthquake, when the seismic energy input can be considered as insignificant. Therefore, it is reasonable to assume the value $\mu_{d}=\mu_{\text {slow }}$ in Equation (5) wherein the force equilibrium under quasi-static condition is considered:

$$
d_{0}=\mu_{\text {slow }} \cdot R_{b}
$$

A possible outcome of an insufficient re-centring capability is the increase in residual and maximum displacements for ground motions with directivity effects, like near-fault earthquakes, where the most of the energy is introduced in the system by a single pulse [2; 32]. For ground motions with such characteristic, the effect of a displacement offset is now examined using energy concepts. The movement of the isolation system from its origin to the maximum displacement $d_{\max }$ is represented in Figure 2 as a solid line (branch $\mathrm{OAB}$ ). Assume now that at the beginning of the earthquake the system is affected by the eccentricity $\Delta d_{i}=$ OO', which could be due to e.g. the residual displacement at the end of a preshock. The offset leads to a corresponding change $\Delta d_{\max }$ in the maximum displacement (branch O'A'B'). As the energy induced by the earthquake is the same for 
the two examined branches, the law of conservation of energy requires the two shaded areas $E_{1}$ and $E_{2}$ to be equal. This implies that the two quantities $\Delta d_{i}$ and $\Delta d_{\max }$ have the same sign and the relation between them is provided by simple geometrical calculations [3]

$$
\Delta d_{\max }=\frac{1}{1+\frac{d_{\max }}{d_{0}}} \Delta d_{i}
$$

In Equation (10) the sign of $\Delta d_{i}$ (and consequently of $\Delta d_{m a x}$ ) is positive when the offset is in the same direction along which the maximum displacement $d_{\max }$ occurs, and negative otherwise (Figures 2(a) and 2(b) respectively). The displacement accrual depends on both the properties of the isolators, expressed by the parameter $d_{0}$, and the details of the ground motion, which affect the magnitude and the direction of the peak displacement $d_{\max }$. As evident from Equation (10), the influence of the offset is expected to be lower for strong earthquakes producing larger motions than for weak earthquakes.

However, it has to be noted that a decrease of the maximum displacement may be also possible. When the isolation is in its origin (position $\mathrm{O}$ ), the inertial force produced by the ground acceleration must overcome the characteristic strength $F_{0}$ before the system is displaced, and owing to the symmetry of the loop (points A and E in Figure 2(a)), the motion has the same likelihood of starting in either direction. On the contrary, when the offset $\Delta d_{i}$ is present, the breakaway resistance is no longer symmetric since the restoring force is always directed towards the origin: the resisting force is (in its absolute value) equal to $F_{0}+K_{\mathrm{P}} \cdot \Delta d_{i}$ for motion in the positive direction of the displacement axis (point $\mathrm{A}^{\prime}$ ), and equal to $F_{0}-K_{\mathrm{P}} \cdot \Delta d_{i}$ (point E') for motion in the opposite direction. Hence, a lower magnitude of ground acceleration will be enough to initiate the motion in the direction opposite to the offset than in the same direction, which entails a decrease in $d_{\max }$ according to Equation (10). Similar conclusions apply to the situation of Figure 2(b), with consideration of the different sign. 
In the above considerations, the dependency of the coefficient of friction on the axial load and on the temperature of the sliding surfaces was not accounted for. In practice, the axial load acting on the individual bearings can undergo considerable variations during the earthquake due to the rocking of the superstructure and the vertical ground excitation, and heating of the sliding surfaces occurs as well following the huge dissipation of seismic energy by means of friction. All these effects contribute to changes in the actual coefficient of friction of the CSS surfaces in addition to the effect of velocity. Though the rigorous conclusion were that the classic bilinear model is not representative of the actual behaviour of the Curved Surface Slider under seismic excitation, it is of practical interest to verify whether the conclusions drawn for the simple bilinear systems are still applicable, at some extent, to describe the basic features of the re-centring behaviour of CSS systems.

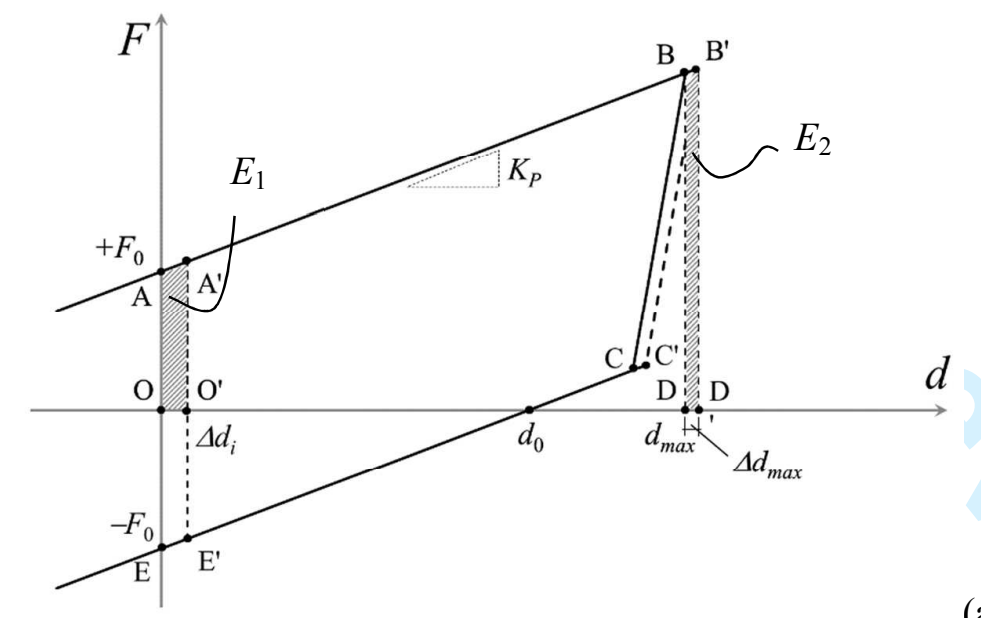

(a)

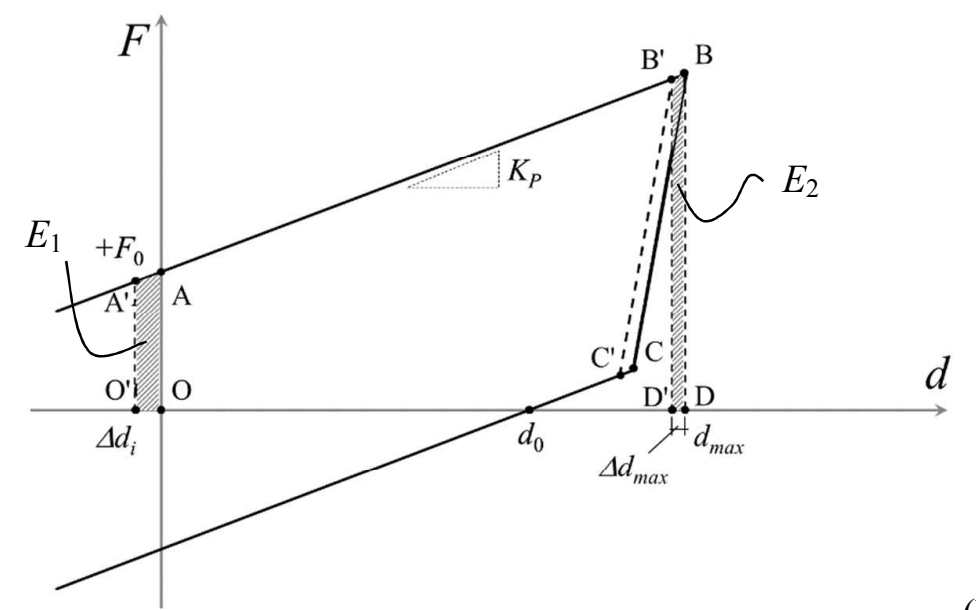

(b) 
1

2

3

4

5

6

7

8

9

10

11

12

13

14

15

16

17

18

19

20

21

22

23

24

25

26

27

28

29

30

31

32

33

34

35

36

37

38

39

40

41

42

43

44

45

46

47

48

49

50

51

52

53

54

55

56

57

58

59

60

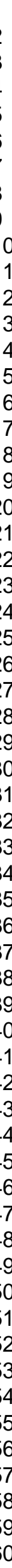

Figure 2. Influence of the initial offset $\Delta d_{i}$ on the maximum displacement $d_{\max }: \Delta d_{i}>0$ and $\Delta d_{\max }>0$ (a); $\Delta d_{i}<0$ and $\Delta d_{\max }<0(\mathrm{~b})$ 


\section{MATERIALS AND METHODS}

\section{Experimental set-up}

The investigation was performed at the laboratory of the Department of Structures for Engineering and Architecture at the University "Federico II" of Naples, Italy. The testing facility was a $3 \times 3 \mathrm{~m}$ biaxial shake table, with maximum payload of $200 \mathrm{kN}$, frequency range of $0-50 \mathrm{~Hz}$, acceleration peak equal to $1 \mathrm{~g}$, velocity peak equal to $1 \mathrm{~m} / \mathrm{s}$ and total displacement capacity of 500 $\mathrm{mm}$.

The mock-up consisted of a four columns, one story steel frame with a rectangular plant of $2.65 \times 2.15 \mathrm{~m}$ span and $2.90 \mathrm{~m}$ total height (Figure 3 ), which could be assumed as representative of a building at one third-length scale. Details of the mock-up can be found elsewhere [33]. Each floor contained additional concrete blocks / slabs to provide a total mass of 8200 tons. The base level has a mass of 3.26 tons, whereas the top floor had a mass of 4.94 tons.

The mock-up was supported by four bearings bolted to the base frame in correspondence of the columns. Owing to the symmetry of the structure, each isolator carried a quarter of the total weight. In order to prevent the structure from dropping off the shake table in case of excessive movements, safety constraints limited the relative displacements to $\pm 125 \mathrm{~mm}$. 


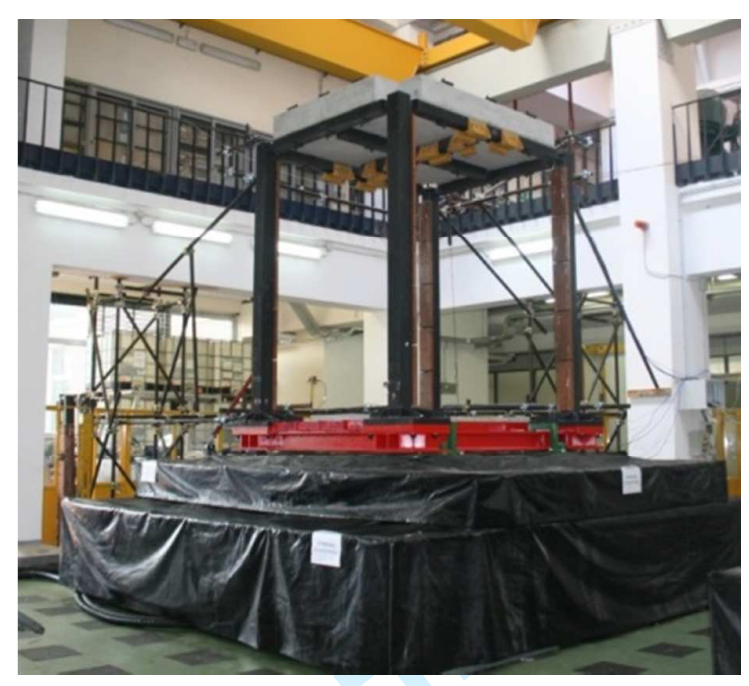

(a)

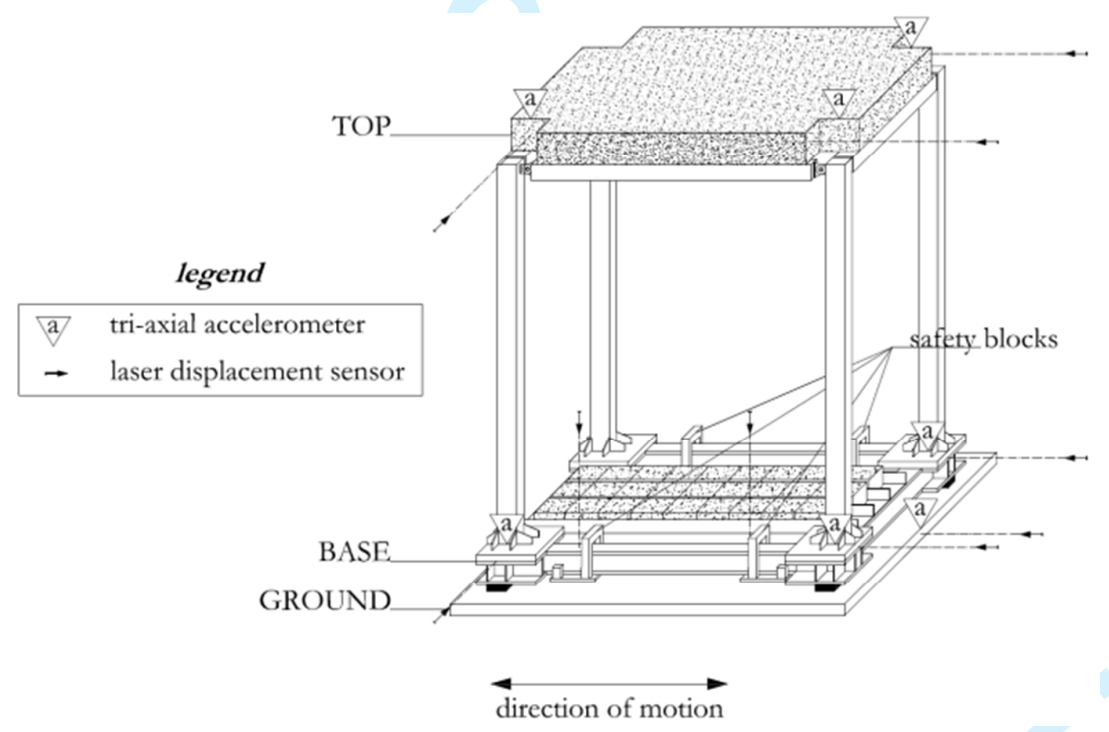

(b)

Figure 3. Test set-up: view of the structure mock-up on the shake table (a); and cabinet projection of the mock-up and the instrumentation set-up (b).

The scale factors of the structural model are summarized in Table 1. The fundamental frequency of the fixed-base model is $2.5 \mathrm{~Hz}$ (i.e. a period $\mathrm{T}=0.4 \mathrm{~s}$ ), which corresponds to a period of the fullscale structure of $0.7 \mathrm{~s}$, common for seven-story buildings. The damping for the fundamental mode is $0.6 \%$, similar to the majority of steel frames in the elastic range with no additional sources of energy dissipation. 
In the experiments, unidirectional acceleration time histories were imposed to the shake table along the direction of maximum span of the mock-up. The absolute displacements of the mock up were monitored by six laser displacement sensors, model CP35MHT80 (Wenglor Sensoric GmbH, Germany), with $\pm 150 \mathrm{~mm}$ capacity and $50 \mu \mathrm{m}$ resolution; at each floor two transducers were used to measure the displacement in the direction of motion, whereas one transducer measured the displacement in the transverse direction for detecting any in-plane rotations. The absolute accelerations of the floor masses were measured by means of six triaxial accelerometers model 356A17 (PCB Piezotronics Inc, NY), with \pm 10 g capacity, placed at the corners of structure, three at each floor. To complete the instrumentation layout, an additional laser transducer was used to measure the absolute displacement of the table in the direction of testing, and an accelerometer recorded the motion of the table to check the actual ground motion transmitted to the structure. Displacements and accelerations measured by the sensors were sampled at $500 \mathrm{~Hz}$ and filtered at 50 Hz. The instrumentation set-up is illustrated in Figure 3(b). 
Table 1. Summary of Scale Factors.

\begin{tabular}{llc}
\hline Variable & Dimension & Scale Factor \\
\hline Linear Dimension & Length & $1 / 3$ \\
Displacement & Length & $1 / 3$ \\
Time & Time & $1 / \sqrt{ } 3$ \\
Mass & Mass & $1 / 9$ \\
Velocity & Length $\cdot$ Time $^{-1}$ & $1 / \sqrt{ } 3$ \\
Acceleration & Length· Time & 1 \\
Frequency & Time $^{-2}$ & $\sqrt{3}$ \\
Force & Mass $^{-2}$ Length $\cdot$ Time $^{-2}$ & $1 / 9$ \\
\hline
\end{tabular}

\section{Curved Surface Sliders}

Curved Surface Slider prototypes were manufactured for the $1 / 3$ scaled model according to two alternative designs depending on the displacement capacity but with same effective radius and restoring stiffness. The first design was a Double Curved Surface Slider (DCSS), Figure 4(a), with upper and lower concave backing plates having identical radii $R_{1}=R_{2}=770 \mathrm{~mm}$, and a rigid slider with height $h=55 \mathrm{~mm}$, providing an effective radius $R_{b}=R_{1}+R_{2}-h=1485 \mathrm{~mm}$ [34]; the displacement capacity was $260 \mathrm{~mm}$. The second design was a Single Curved Surface Slider (SCSS), Figure 4(b), with radii $R_{1}=1270 \mathrm{~mm}$ and $R_{2}=300 \mathrm{~mm}$, respectively, and a rigid slider with height $h=70 \mathrm{~mm}$, providing an effective radius $R_{b}=1500 \mathrm{~mm}$; the displacement capacity was $165 \mathrm{~mm}$.

Four SCSS bearings and four DCSS bearings were produced and assembled. The backing plates and the sliders of the bearings were made of S355 structural steel, with the concave sliding surfaces lined with a $2.5 \mathrm{~mm}$ thick stainless steel sheet. The sliding pads had a diameter of either $80 \mathrm{~mm}$ (SCSS bearing) or $60 \mathrm{~mm}$ (DCSS bearing) and a thickness of $6 \mathrm{~mm}$. A rotation pad made of 
lubricated PTFE was used in the spherical joint (curved surfaces with radius $R_{2}$ ) of the SCSS bearings.

Three different materials were used for the sliding pads:

- standard PTFE (polytetrafluoroethylene) resing lubricated with silicon grease (L-TF);

- composite material made of PTFE resin with metal fillers, lubricated with silicon grease (L-CM);

- composite material made of PTFE resin with metal fillers, not lubricated (NL-CM).

L-TF and L-CM pads were used in the DCSS bearings, whereas NL-CM pads in the SCSS bearings.

The coefficient of friction of the three materials material was characterised in small scale tests conducted at the laboratory of Politecnico di Milano in accordance with the experimental procedure described elsewhere [25]. The material specimens were tested under the application of a constant pressure of either $7.10 \mathrm{MPa}$ (L-TF and L-CM materials) or $3.98 \mathrm{MPa}$ (NL-CM material), corresponding to the actual load acting on the pads when installed the CSS bearings due to the weight of the mock-up. The experimental data obtained in the tests were curve-fitted in accordance with Equation (8), providing the values of the $\mu_{\text {slow }}, \mu_{\text {fast }}$ and $\alpha$ parameters reported in Table 2.

Table 2. Parameters of the friction law of the pad materials and relevant test pressure $p$ (ambient temperature, velocity range: 1 to $200 \mathrm{~mm} / \mathrm{s})$.

\begin{tabular}{ccccc}
\hline Material & pressure, MPa & $\mu_{\text {slow }}$ & $\mu_{\text {high }}$ & $\alpha, \mathrm{s} / \mathrm{mm}$ \\
\hline L-TF & 7.10 & 0.008 & 0.039 & 0.003 \\
L-CM & 7.10 & 0.022 & 0.100 & 0.002 \\
NL-CM & 3.98 & 0.032 & 0.265 & 0.002 \\
\hline
\end{tabular}




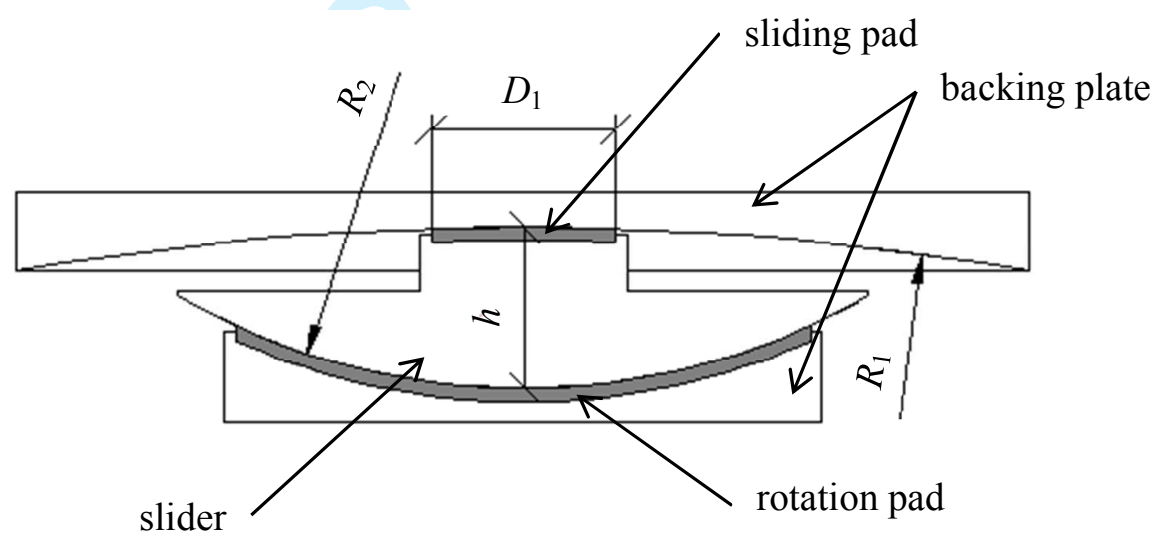

Figure 4. Geometrical parameters of Double Curved Surface Slider (DCSS) prototype (a), and Single Curved Surface Slider (SCSS) prototype (b)

\section{Characterization of the isolation system}

Preliminary to the seismic tests, the dynamic properties of the isolation system were characterized by means of sine sweep excitation tests conducted on the isolated mock-up. A displacement amplitude of $50 \mathrm{~mm}$ was imposed to the table motion with frequency varying from 0.25 to $1.5 \mathrm{~Hz}$ with an incremental step of $0.05 \mathrm{~Hz}$. Three displacement cycles at every frequency level were carried out. Table 3 reports for the different configurations the fundamental frequency $f_{\text {iso }}$ of the base isolated structure obtained by Fast Fourier Transforms (FFTs) of the roof acceleration signals, the corresponding fundamental period $T_{\text {iso }}$, and the properties of the isolation system at $f_{\text {iso }}$. 
Table 3. Fundamental properties of the isolation system and corresponding dynamic properties.

\begin{tabular}{lllllll}
\hline Bearing model & Sliding pad & $f_{\text {iso }}, \mathrm{Hz}$ & $T_{\text {iso }}, \mathrm{s}$ & $\xi_{\text {eq }},-$ & $F_{0}, \mathrm{kN}$ & $K_{P}, \mathrm{kN} / \mathrm{m}$ \\
\hline DCSS & L-TF & 0.55 & 1.82 & 28.5 & 0.603 & 13.54 \\
DCSS & L-CM & 1.06 & 0.94 & 54.2 & 1.608 & 13.54 \\
SCSS & NL-CM & 1.16 & 0.86 & 55.8 & 2.412 & 13.41 \\
\hline
\end{tabular}

\section{Ground motion records}

Seven independent ground motion records consistent with the current Italian Building Code [35] were selected from the European Strong-motion Database [36] using REXEL v3.4 beta software [37]. The selected horizontal acceleration histories are in compliance with the assumed code for the life safety limit state of a strategic structure (functional class IV) located in Naples, Italy (14.2767 longitude, $40.863^{\circ}$ latitude) on soil type A (stiff soil or rock) with a nominal life of 100 years (corresponding to a 1898-year return period according to the code). Only events in the magnitude $(\mathrm{Mw})$ interval $[5.3,7.3]$ and with an epicentral distance $(R)$ interval $[0-80 \mathrm{~km}]$ were considered, which reflects the hazard disaggregation for the spectral acceleration $\mathrm{Sa}(\mathrm{T})$ for the period of interest in the nonlinear structural behaviour. This selection is representative of regions in Italy with a moderate to high seismic risk. The selected waveforms were compressed in time by a factor of $\sqrt{ } 3$ to satisfy the similitude requirements and scaled to the Peak Ground Acceleration (PGA) level of 0.259 g, which corresponds to the PGA value of the site according to the code assuming a topographic category T1. Scale Factors (SFs) of the acceleration time histories ranged from 0.73 to 1.43 . The selected ground motions are listed in Table 4 and the scaled 5\% damped elastic spectra are illustrated in Figure 5. 


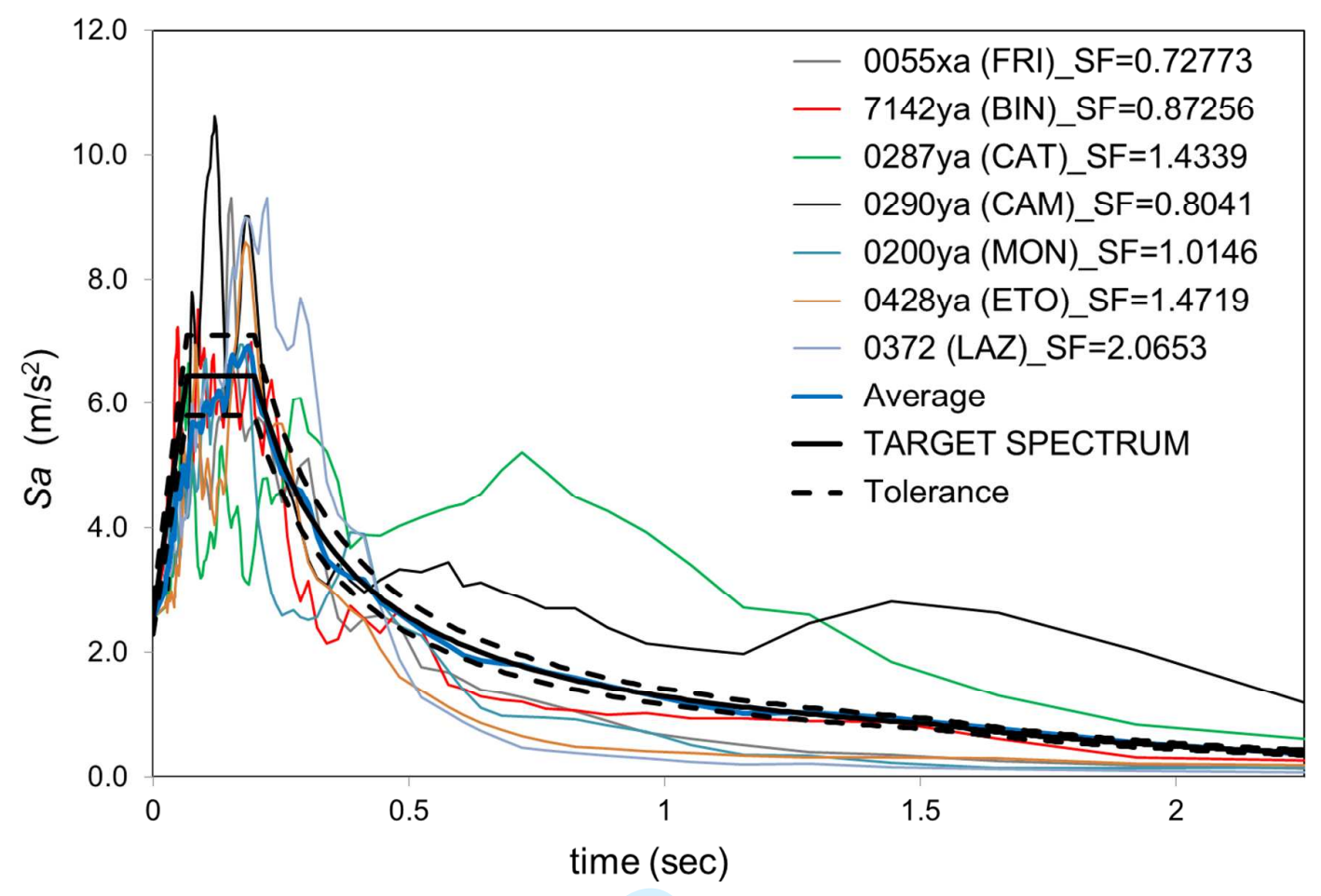

Figure 5. Scaled ground motion spectra and target spectrum according to Italian Building Code $\left(S_{T}=1 / 1 / \sqrt{3} ; \mathrm{SF}_{\text {mean }}=\right.$ $1.1981)$ 
Table 4. Selected ground motions and details.

scaled earthquake

\begin{tabular}{|c|c|c|c|c|c|c|c|c|c|c|}
\hline Record & & Waveform ID & Station ID & Date, $\mathrm{dd} / \mathrm{mm} / \mathrm{yy}$ & $\mathrm{SF}$ & Mw & $R, \mathrm{~km}$ & PGA, $\mathrm{m} / \mathrm{s}^{2}$ & $\mathrm{PGV}, \mathrm{cm} / \mathrm{s}$ & PGD, cm \\
\hline Bingol & $(\mathrm{BIN})$ & 7142 ya & ST539 & $01 / 05 / 2003$ & 0.87 & 6.3 & 14 & 2.55 & 18.29 & 3.25 \\
\hline Friuli & (FRI) & $55 \mathrm{xa}$ & ST20 & 06/05/1976 & 0.72 & 6.5 & 23 & 2.55 & 15.25 & 9.29 \\
\hline Montenegro & $(\mathrm{MON})$ & $200 y a$ & ST68 & $15 / 04 / 1979$ & 1.01 & 6.9 & 65 & 2.55 & 12.87 & 9.60 \\
\hline Etolia & (ETO) & 428ya & ST169 & $18 / 05 / 1988$ & 1.47 & 5.3 & 23 & 2.55 & 12.46 & 6.06 \\
\hline Lazio Abruzzo & (LAZ) & $372 y a$ & ST274 & 07/05/1984 & 2.06 & 5.9 & 68 & 2.55 & 15.02 & 6.80 \\
\hline Campano Lucano & (CAM) & $290 y a$ & ST96 & $23 / 11 / 1980$ & 0.80 & 6.9 & 32 & 2.55 & 44.10 & 16.20 \\
\hline Campano Lucano & $(\mathrm{CAT})$ & $287 y a$ & ST93 & $23 / 11 / 1980$ & 1.43 & 6.9 & 23 & 2.55 & 43.90 & 14.00 \\
\hline mean & & & & & 1.19 & 6.4 & 35 & & & \\
\hline
\end{tabular}




\section{Shake table tests}

Two protocols were performed within the present investigation.

The first, or "single event", experimental protocol aimed at verifying the restoring capability of the isolation system comprised of low dissipation Curved Surface Sliders. The mock-up was isolated with four CSS bearings with L-TF pads and subjected to the series of the seven one-directional ground motion records listed in Table 5. Each record was run once. The isolators were centred at the beginning of the series and if necessary in case of substantial residual displacement. Because of the huge displacements (restrained by the safety blocks) produced by the 290ya waveform, the record was scaled to $50 \%$ of the reference PGA.

The second, or "motion sequence", experimental protocol aimed at investigating the accrual of displacements due to an initial offset. The protocol was performed on the three configurations of the isolation system assessed in the characterization tests (Table 3). The detail of the ground motion sequences is given in Table 5: the BIN (7142ya), the CAT (287ya), and the FRI (55xa) records were run three to five times each; the CAM (290ya) record was run five times only for the configuration with high friction NL-CM pads. The isolators were in undeformed configuration before the beginning of the sequences. The possible residual displacement at the end of each run of ground motion was not removed, but remained as the initial offset at the beginning of the next run. Assuming that the input energy was identical in each run of the same ground motion record, the dependence of the maximum displacement on the initial offset was appraised. 
Table 5. Test sequence of ground motions: order of events and number of runs

\begin{tabular}{cccc}
\hline $\begin{array}{c}\text { Ground motion } \\
\text { record }\end{array}$ & L-TF & L-CM & NL-CM \\
\hline BIN & 3 & 5 & 5 \\
CAT & 3 & 5 & 5 \\
FRI & 3 & 3 & 5 \\
CAM & $=$ & $=$ & 5 \\
\hline
\end{tabular}

The response of the isolated structure was assessed in terms of relative displacements respect to the shake table and of absolute accelerations. The relative displacements were obtained by subtracting the shake table motion from the absolute displacements measured by the laser sensors fixed to the external frame. At each floor, the displacement of the structure in the direction of motion was calculated by averaging the displacements measured at two corners, see Figure 3(b). Absolute accelerations were directly measured by the accelerometers.

As an example, Figure 6 illustrates the time histories of the ground acceleration measured by the accelerometer on the shake table and the mock-up displacement at the base level during a run of the CAT (287ya) ground motion record for the three CSS configurations. The effect of the coefficient of friction in limiting the structure displacements through the mechanism of energy dissipation but at the same time reducing the re-centring capability is evident: the residual displacement is insignificant with low friction sliders (L-TF pads), small with medium friction sliders (L-CM pads) whereas it is a significant amount of the maximum displacement with high friction sliders (NL-CM pads). 

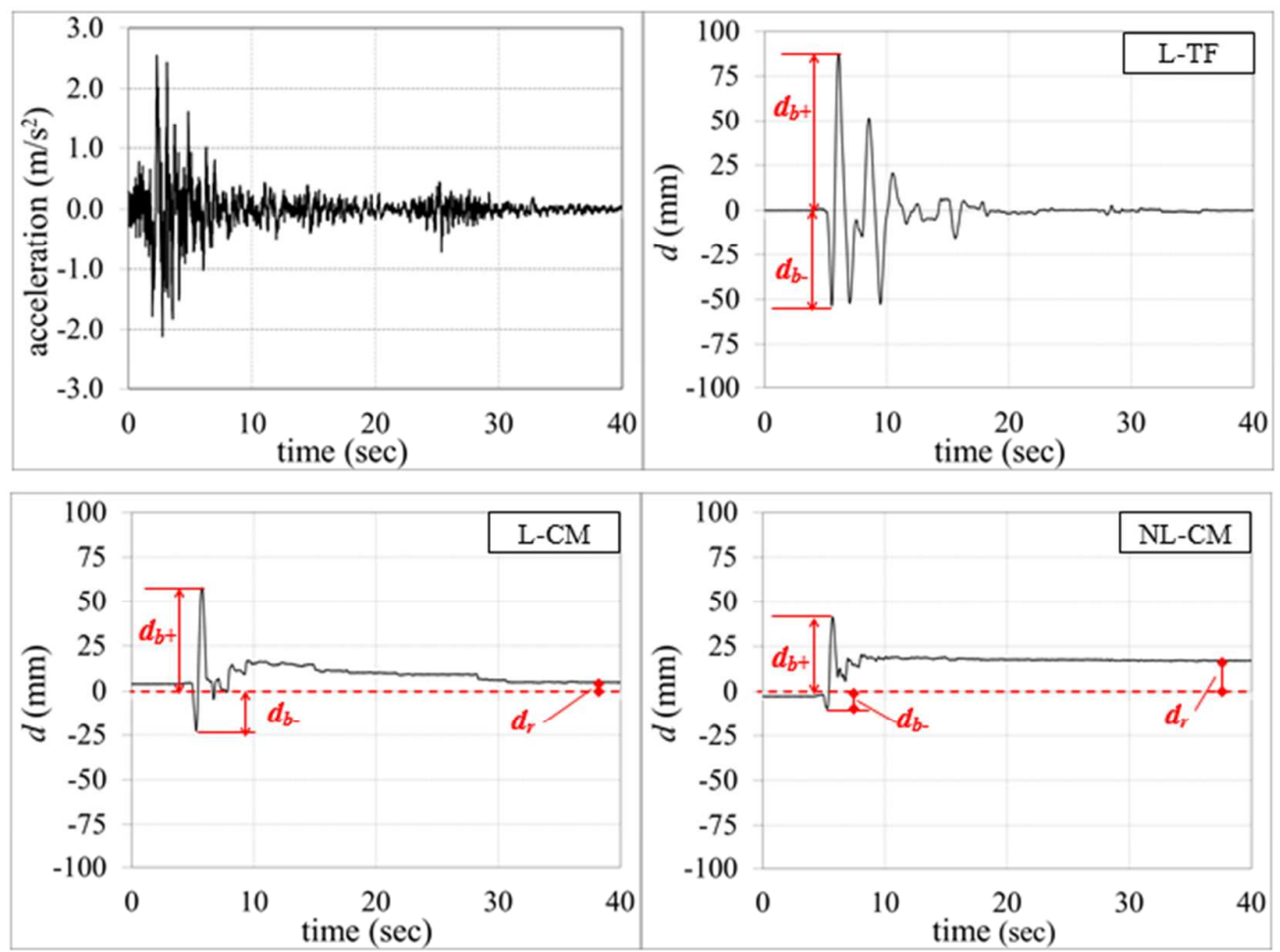

Figure 6. Time histories of shake table acceleration and structure base displacement during a run of CAT (287ya) ground motion record 


\section{Results}

The results are analysed accounting for the following quantities: the peak displacements $d_{b^{+}}, d_{b-}$ of the structure at the isolation base level in either direction of motion, the maximum displacement $d_{\max }$ $=\max \left(d_{b^{+}},\left|d_{b_{-}}\right|\right)$, the residual displacement $d_{r}$, and the peak absolute acceleration $\left(a_{\max }\right)$ at the top floor of the structure. $d_{b^{+}}, d_{b^{+}}$and $d_{r}$ are in Figure 6.

The results of the "single event" protocol are shown in Figure 7. Assuming the largest maximum displacement over the seven ground motions $\left(d_{\max }=88.7 \mathrm{~mm}\right.$, CAT record $)$ as the design displacement $d_{c d}$ of the isolation system, for the CSS configuration with L-TF pads the ratio $d_{c d} / d_{0}$ results equal to $7.38\left(\mu_{\text {slow }}=0.008\right.$ as given in Table 2$)$. The residual displacement after a single earthquake becomes smaller with the increasing of the maximum displacement $d_{\max }$, and the largest residual over the seven independent ground motions $(3.5 \mathrm{~mm}$, CAM record) is less than $4 \%$ of the assumed $d_{c d}$.

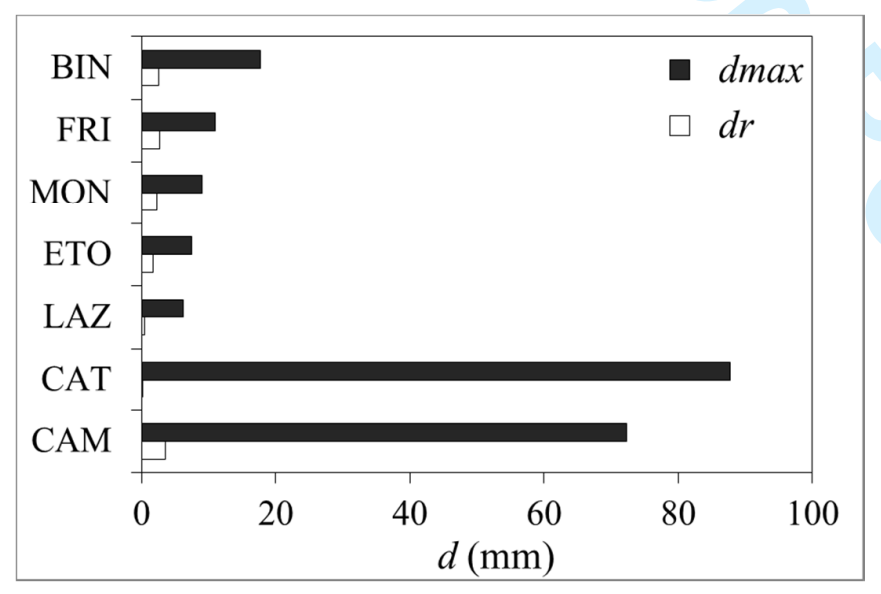

Figure 7. Maximum and residual absolute displacements of the CSS isolation system with low friction (L-TF) pads over seven spectrum-compliant ground motions. 
For the analysis of the results of the "motion sequence" protocol, the design displacement $d_{c d}$ of the isolation system was assumed as the displacement recorded at the first run of the 287ya (CAT) ground motion. As shown in Table 6, the code requirement $d_{c d} / d_{0}>0.5$ [17] was always fulfilled (relevant level of $\mu_{\text {slow }}$ as given in Table 2).

Table 6. Evaluation of the re-centring capability of the CSS isolation system according to the Eurocode 8 depending on the friction coefficient of the sliders

\begin{tabular}{cccc}
\hline sliding pad & $d_{c d}, \mathrm{~mm}$ & $d_{0}, \mathrm{~mm}$ & $d_{c d} / d_{0}$ \\
\hline L-TF & 87.7 & 11.9 & 7.38 \\
L-CM & 61.3 & 32.7 & 1.88 \\
NL-CM & 41.2 & 48.0 & 0.86 \\
\hline
\end{tabular}

Peak and residual displacement time-histories of the test sequences are illustrated in Figure 8, which reports, for every run of ground motion, the peak displacements $d_{b+}, d_{b \text {-, }}$ the initial offset $d_{i}$ and the residual displacement $d_{r}$ (the initial offset at the beginning of the next run).

The accrual of residual displacements is not significant for the CSS system with L-TF pads (Figure 8(a)).

Enough re-centring capability is shown also by the Curved Surface Sliders with L-CM pads (Figure $8(\mathrm{~b})$ ). The small offset at the beginning of the sequence of CAT ground motion remains unchanged during the whole sequence. The offset at the beginning of the FRI sequence is progressively recovered during three runs of the ground motion, with a corresponding decrease of the maximum displacement in each run.

On the contrary, insufficient re-centring capability is demonstrated by the CSS system with NLCM pads under the CAM and CAT sequences (only the displacement time-histories during the first sequence are reported due to similar trends), as reflected by the displacement growth (Figure 8(c)). 
At the end of the first run of ground motion a residual deformation occurs, which further increases at the end of the second and of the third run. The same behaviour is reflected by the maximum displacement of the system; however, the displacement build-up seems to end after the third run (Figure 9(a)). On the contrary, at the end of each run of the FRI ground motion sequence the system progressively returns towards its origin (Figure 9(b)) involving a decrease in the displacement amplitude; this behaviour is justified by Equation (10) as $\Delta d_{i}$ and $d_{\max }$ have opposite signs. 

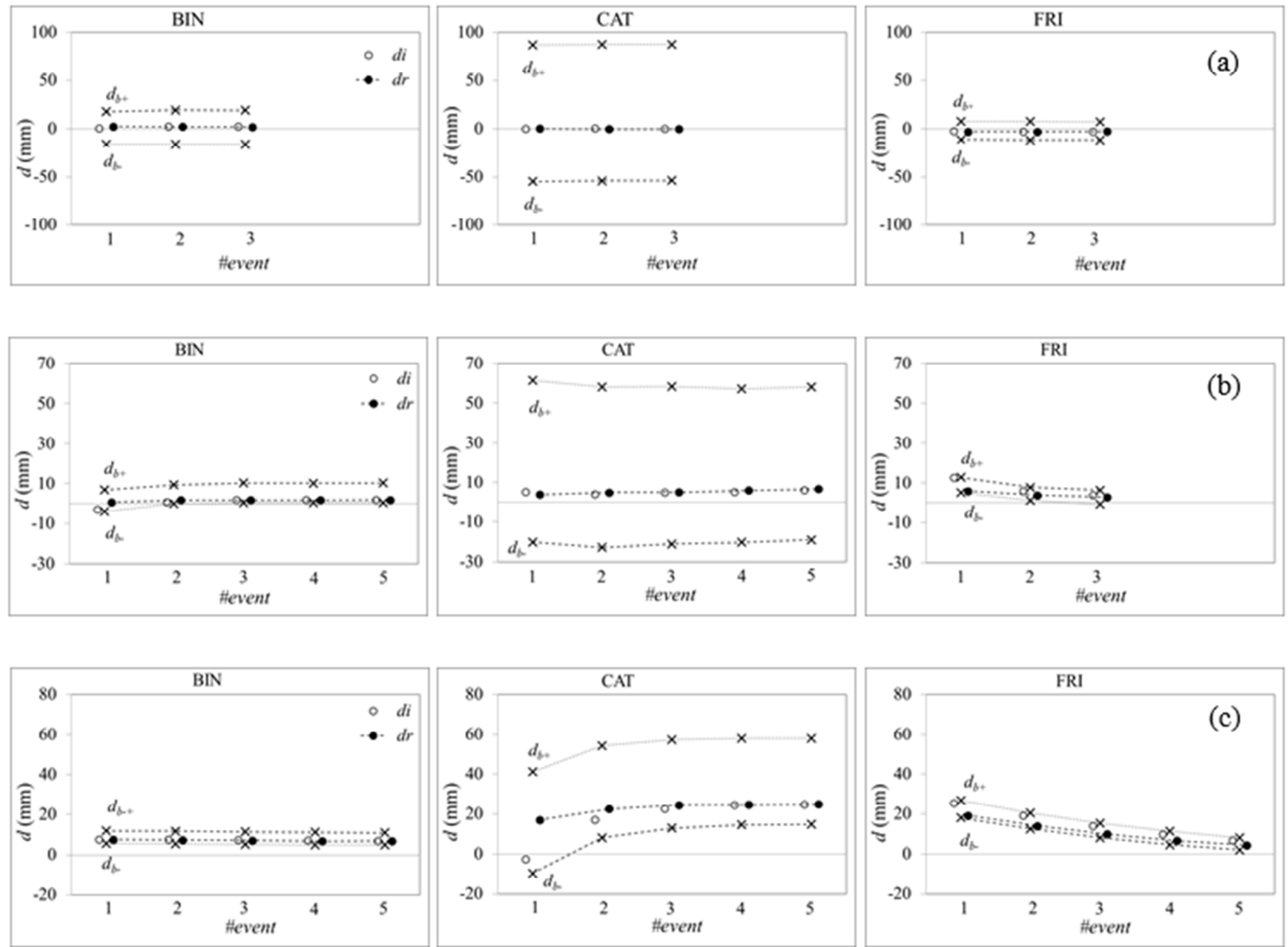

Figure 8. Peak displacement in either direction of motion $\left(d_{b^{+}}, d_{b_{-}}\right)$and residual displacement $\left(d_{r}\right)$ depending on the initial offset $\left(d_{i}\right)$ during sequences of identical ground motion records: CSS system with L-TF pads (a), L-CM pads (b), and NL-CM pads (c).

Finally, Figure 10 shows the acceleration amplification factor, i.e. the ratio of the peak acceleration at the top floor of the structure to the peak ground acceleration. The largest accelerations occur for the isolators with high friction NL-CM pads, but remain nearly constant throughout each ground motion sequence, notwithstanding either the growth or decrease of permanent deformation. At least for the considered ground motion time histories, the acceleration amplification is not sensitive to the initial eccentricity of the isolation system, nor to the displacement accrual, and this result seems to be independent on the re-centring capability of the isolators. 

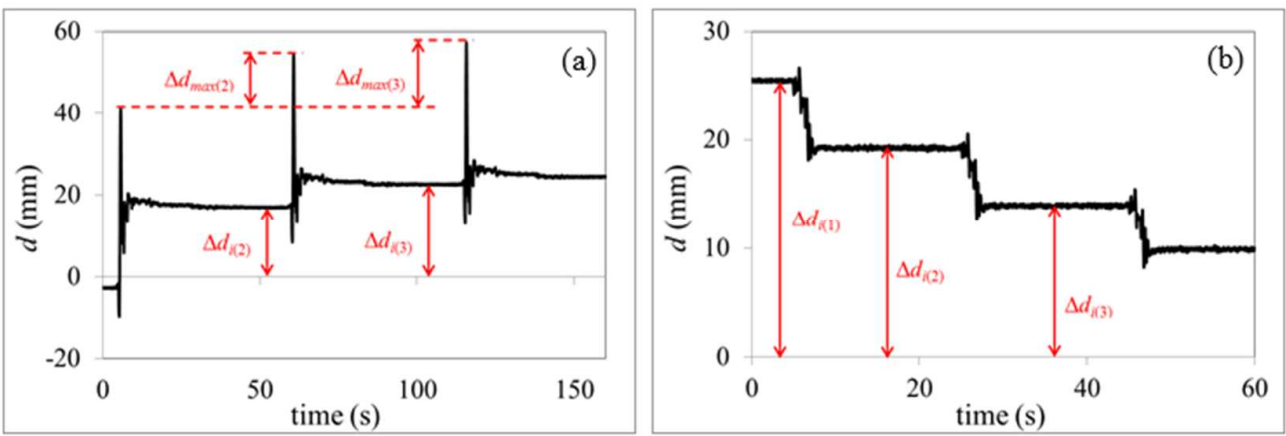

Figure 9. Influence of the ground motion details on the accrual of maximum and residual displacement: displacements build up when the maximum displacement occurs in the same direction of the offset (a); re-centring occurs when the maximum absolute displacement occurs in the opposite direction (b)
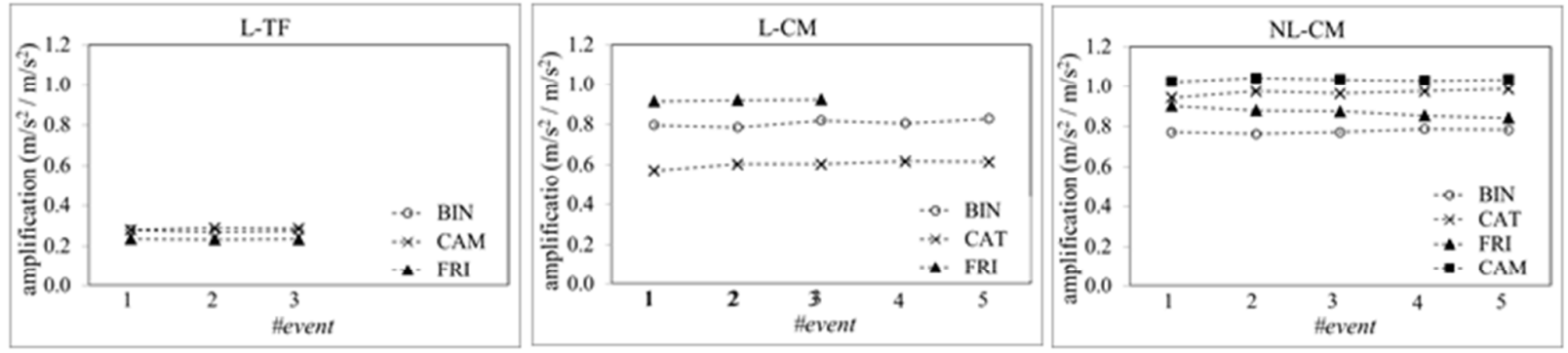

Figure 10. Acceleration amplification at the top floor of the structure 


\section{DISCUSSION}

Studies on the re-centring capability of bilinear hysteretic systems had been performed in the past [3], under the implicit assumption that the properties of the system do not change during the seismic event. Similar analyses were the basis for the re-centring criteria formulated in current codes [17]. It is therefore an argument whether or not the codes' provisions are valid for the Curved Surface Slider, because of the variability of the coefficient of friction during operation depending on the axial load, the velocity and the temperature.

To comply with the available testing facility, the present study was conducted on a one-third scaled structural mock-up of about $8000 \mathrm{~kg}$ mass, under the application of uni-directional ground motion time histories. The isolating devices were scaled to respect the geometric similitude requirements, but due to the strong nonlinearities introduced by friction, it is questionable how much the response of the scaled prototypes reflects the behaviour of real-scale isolators [38]. The unidirectional excitation represents another important simplification since, during the earthquake, isolation devices are in general subjected to the effect of general three-dimensional acceleration time histories. Biaxial tests performed on sliding isolators with curved surfaces $[39,40]$ proved the nonnegligible effects of the two horizontal components of motion that increase the heating of the sliding surfaces, leading to lower friction and consequently damping. These latter effects are expected to foster the re-centring of the devices, because result in increased displacement amplitude $d_{\max }$ and smaller value of $d_{0}$. Intuitively, also the vertical excitation of earthquakes should affect the horizontal response of a structure isolated with curved surface sliders since the horizontal force is proportional to the instantaneous vertical force. However, the experimental evidence seems to confirm that the coupling phenomenon between horizontal and vertical excitation can influence the horizontal response of the isolated structure in terms of shear forces, whereas it has little influence on the horizontal displacement [41-44]. 
The investigation presented in the study, though affected by the above limitations, has is the merit of being, to the knowledge of the authors, one of the first experimental studies addressing the effect of the coefficient of friction on the restoring capability of Curved Surface Sliders and the possible displacement accrual under a sequence of shakes.

The shake table tests point to the fact that also for the CSS system the re-centring capability improves as the parameter $d_{c d} / d_{0}$ increases, but the provision $\delta=0.5$ recommended by the code [17] seems to be not conservative for isolators with high friction materials, as the friction force can effectively contrast the re-centring action:

(a) The CSS isolation system with either low or medium friction coefficient demonstrated inherent re-centring capability, with small residual displacements in comparison to the design level $\left(d_{r} / d_{c d}\right.$ less than 0.04 and less than 0.10 respectively), and a not significant increase in the maximum deformation regardless of the initial eccentricity $\Delta d_{i}$; this result is in agreement with the recentring criterion of the Eurocode 8 based on the requirement $d_{c d} / d_{0} \geq 0.5$;

(b) The CSS isolation system with high friction, though meeting the code requirement $\left(d_{c d} / d_{0}=\right.$ 0.86), demonstrated an insufficient restoring behaviour: the permanent displacement can be an important part of the design displacement, possibly affecting the entity of the maximum displacement in a future event: e.g. at the end of the CAT ground motion sequence the permanent displacement was more than $50 \%$ of the maximum displacement $\left(d_{r}=0.56 d_{c d}\right)$, and the displacement amplitude increase of about $40 \%$ respect to the value at the first shacking .

Though the number of CSS configurations (only one effective radius $R_{b}$ was investigated) and ground motion time histories considered in the study is very limited and a generalization of the results to different situations cannot be made, some considerations can be drawn. First, the build-up of residual displacements always ended even in the unfavourable event in which the residual displacement of each earthquake was additive to the accumulated residual displacement of the previous earthquakes. Second, the peak displacement in either direction of motion, and hence the 
maximum displacement $d_{\max }$ during the earthquake, is influenced by the initial eccentricity $d_{i}$. An offset from the origin $\Delta d_{i}$ produces variations $\Delta d_{b+}$ and $\Delta d_{b-}$ that have the same sign of $\Delta d_{i}$ so that the displacement time history of the isolation system is shifted in the same direction of the eccentricity, and the larger $\Delta d_{i}$, the larger $\Delta d_{\max }$; this is illustrated in Figure 9 and is in agreement with Equation (10). Nevertheless, in some situations the ground motion may have a restoring effect and tends to re-centre the isolation system (e.g. Figure 9(b)). In section 2 it was explained that this is likely to occur especially for low intensity earthquakes owing to the contribution of the restoring force $K_{\mathrm{P}} \cdot \Delta d_{i}$, always directed towards the origin, to the resisting force at breakaway. This confirms that the re-centring capability does not depend on the mechanical characteristics of the isolation system alone, but also on the details of the seismic waveform.

According to the codes, the displacement demand is calculated based on the single occurrence of the design earthquake, and without consideration of any initial offset. For Curved Surface Sliders with good re-centring capability the displacement demand does not change significantly even in the event of a seismic sequence comprising pre-shocks or aftershocks. But for systems with low selfcentring capability large residual displacement can occur under this circumstance and possibly jeopardize the displacement capacity of the device if the likelihood of displacement accrual was not adequately accounted for during their design.

It is worth noting that some practical issues have to be considered which require a more prudential estimate of the re-centring capability. First, the coefficient of friction tends to increase with wear and contamination of the sliding surfaces, like e.g. in case of inadequate protection from dust, and consequently the re-centring capability must reasonably be expected to decrease during the lifetime of CSSs. Also the "stick slip" phenomenon can lead to a dramatic decrease in the restoring behaviour of the friction devices. Eventually, the presence of an internal articulation in the curved surface slider that allows independent movement of the sliding surfaces from each other can make unpredictable the restoring behaviour of the system. The tests presented in the study were performed on prototypes 
with only two pairs of curved sliding surfaces and a rigid slider, so due to kinematic constraints the two surfaces slide at the same time. Sliders with more than two pairs of sliding surfaces and/or an internal articulation can exhibit delays in either the beginning or stop of sliding of one the sliding surfaces, due e.g. to differences in the coefficient of friction at either surface. It is therefore proposed that a more conservative figure for the criterion $d_{c d} / d_{0}$ should be adopted for Curved Surface Slider isolators.

Though beyond the scope of the paper, it has to be remarked that the final evaluation of the recentring capability of current Curved Surface Sliders shall be performed in shake table tests on a building at the real scale, with real scale isolators and under the application of three dimensional excitations (along the two horizontal and the vertical directions) and possibly full scale real ground motion records. To the knowledge of the authors, the largest shake table is available at the Hyogo Earthquake Engineering Research Centre, otherwise known as E-Defense, of the National Research Institute of Earth Science and Disaster Prevention $[45,46]$. The shake table has a payload of $12 \mathrm{MN}$ and was used to perform tests on a full-scale $2 \times 2$ bay, 5-story moment frame building with dimensions $10 \times 12 \times 16 \mathrm{~mm}$ (width $\times$ length $\times$ height) isolated with triple friction pendulum bearings [41]. 


\section{Conclusions}

The re-centring capability of three isolation systems comprised of Curved Surface Sliders with same restoring stiffness but differing for the coefficient of friction was assessed in shake table tests on a scaled model of a building frame. The results of the tests can be summarized in the next points:

(1) the parameter $d_{c d} / d_{0}$ seems to govern the re-centring capability of the CSS system in accordance with the provisions stipulated in the Eurocode 8 [17]; however the criterion $d_{c d} / d_{0} \geq 0.5$ appears to be not conservative, especially for high friction isolators;

(2) since $d_{c d}$ includes the effects of seismic excitation, the re-centring capability of the Curved Surface Slider depends on the ground motion characteristics, resulting generally better for earthquakes promoting larger maximum displacements;

(3) an initial offset of the isolation system affects the maximum displacement occurring during the earthquake, but the effect is lower for systems with good re-centring capability; the quantities $\Delta d_{i}$ (initial eccentricity or offset of the system) and $\Delta d_{\max }$ (variation of maximum seismic displacement consequent to the offset) have the same sign and are related through the parameter $d_{c d} / d_{0}$

(4) depending on the characteristics of the waveform, the ground motion may either produce an accrual of displacement, or have a restoring effect on a displaced system, reducing the eccentricity at the end of the shaking;

(5) a conservative estimation of the displacement capacity of isolation systems with high friction is highly recommended, since when the design displacement is close to the displacement capacity of the isolators, a not negligible residual displacement may jeopardize the displacement capacity of the isolation system considering the occurrence of possible aftershocks and future earthquakes. Though not directly investigated, attention was also addressed to the fact that the wear and contamination of the sliding surfaces, the use of sliding materials with "stick-slip" behaviour, and the possible delays at the beginning of motion between the sliding surfaces, which are not predictable at 
the design stage, can possibly adversely affect the restoring capability of the Curved Surface Slider, requiring a more conservative re-centring criterion than recommended in the code.

Due to the small number of waveforms considered in the study, the influence of the ground motion characteristics was not investigated in detail, and the conclusions cannot be generalized. Nevertheless the study is of some value in that it is among the first experimental works addressing the influence of the coefficient of friction and the possible effect of a permanent deformation on the displacement accrual, and provides some pieces of factual information on the re-centring behaviour of curved surface sliding isolation systems which can be the basis for the development of more refined theoretical models.

\section{Conflict of Interests}

The authors declare that there is no conflict of interests regarding the publication of this paper.

\section{Acknowledgements}

The authors acknowledge the support provided by the Italian Department of Civil Protection (DPC) in the frame of the national Research Project DPC - ReLUIS (National Network of Laboratories of Seismic Engineering) 2014-2018, Line 6 - Isolation and Dissipation. 


\section{REFERENCES}

[1] Medeot R. Re-centring capability evaluation of seismic isolation systems based on energy concepts. Proceedings of the 13th World Conference on Earthquake Engineering 2004, paper 3106, Vancouver, Canada.

[2] Berton S, Infanti S, Castellano MG, Hikosaka H. Self-centring capacity of seismic isolation systems. Structural Control and Health Monitoring 2006; 4(6), pp. 895-914, DOI: 10.1002/stc.188.

[3] Katsaras CP, Panagiotakos TB, Kolias B. Restoring capability of bilinear hysteretic seismic isolation systems. Earthquake Engineering and Structural Dynamics 2008; 37(4), pp. 557-575, DOI: 10.1002/eqe.772.

[4] ASCE/SEI 7 (revision for 2016) Minimum design loads for buildings and other structures, American Society of Civil Engineers, 1801 Alexander Bell Drive Reston, VA 20191.q

[5] Decanini L, Gavarini C, Mollaioli F. Some remarks on the Umbria-Marche earthquakes of 1997. European Earthquake Engineering 2000; 3, pp. 18-48.

[6] Huang Y, Wu JP, Zhang TZ, Zhang DN. Relocation of the M 8.0 Wenchuan earthquake and its aftershock sequence. Science in China (Series D) 2008; 51(12), pp. 1703-1711, DOI: 10.1007/s11430-008-0135-z.

[7] Di Sarno L, Elnashai AS, Manfredi G. Assessment of RC columns subjected to horizontal and vertical ground motions recorded during the 2009 L'Aquila (Italy) earthquake. Engineering Structures 2011; 33(5), pp. 15141535, DOI:10.1016/j.engstruct.2011.01.023.

[8] Di Sarno L, Yenidogan C, Erdik M. Field evidence and numerical investigation of the Mw $=7.1$ October 23 Van, Tabanli and the $\mathrm{Mw}>5.7$ November Earthquakes of 2011. Bulletin of Earthquake Engineering, 2013; 11(1), pp. 313-346, DOI: 10.1007/s10518-012-9417-0.

[9] Motosaka M, Mitsuji K. Building damage during the 2011 off the Pacific coast of Tohoku Earthquake. Soils and Foundations 2012; 52(5), pp. 929-944, DOI:10.1016/j.sandf.2012.11.012.

[10] Carydis P, Castiglioni C, Lekkas E, Kostaki I, Lebesis N, Drei A. The Emilia Romagna, May 2012 earthquake sequence. The influence of the vertical earthquake component and related geoscientific and engineering aspects. Ingegneria Sismica - International Journal of Earthquake Engineering 2012; XXIX, pp. 31-58.

[11] Riddell R, Newmark NM. Statistical analysis of the response of nonlinear systems subjected to earthquakes. Research Report, University of Illinois at Urbana, 1979.

[12] Mahin SA, Bertero VV. An evaluation of inelastic seismic response spectra. Journal of Structures 1981; 107(9), pp. $1777-1795$.

[13] Kawashima K, MacRae GA, Hoshikuma J, Kazuhiro N. Residual displacement response spectrum. Journal of Structural Engineering 1998; 124(5), pp. 523-530, DOI: 10.1061/(ASCE)0733-9445(1998)124:5(523). 
[14] MacRae GA, Kawashima K. Post-earthquake residual displacements of bilinear oscillators. Earthquake Engineering and Structural Dynamics 1997; 26(7), pp. 701-716, DOI: 10.1002/(SICI)10969845(199707)26:7<701::AID-EQE671>3.0.CO;2-I.

[15] Tsopelas P, Constantinou MC. NCEER - TAISEI corporation research program on sliding seismic isolation systems for bridges - experimental and analytical study of a system consisting of sliding bearings and fluid restoring force/damping devices. Technical Report NCEER-94-0014, National Center for Earthquake Engineering Research, 1994.

[16] Naeim F, Kelly JM. Design of seismic isolated structures. Wiley, 1999.

[17] CEN Eurocode 8: design of structures for earthquake resistance-Part 2: Bridges, EN1998-2:2005+A1:2011. European Committee for Standardization, Bruxelles, Belgium, 2005.

[18] CEN EN 15129: Anti-Seismic Devices. European Committee for Standardization, Bruxelles, Belgium, 2009.

[19] Zayas VA, Low SS, Mahin SA. The FPS earthquake protection system: experimental report. Report No. UCB/EERC-87/01, Earthquake Engineering Research Center, University of California at Berkeley, 1987.

[20] Zayas VA, Low SS, Mahin SA. A simple pendulum technique for achieving seismic isolation. Earthquake Spectra, 1990; 6(2), pp. 317-333, DOI: 10.1193/1.1585573.

[21] Mokha AS, Constantinou MC, Reinhhorn AM. Experimental study of friction-pendulum isolation system. Journal of Structural Engineering 1991; 117(4), pp. 1201-1217, DOI: 10.1061/(ASCE)0733-9445(1991)117:4(1201).

[22] Constantinou MC, Whittaker AS, Kalpakidis Y, Fenz DM, Warn GP. Performance of seismic isolation hardware under service and seismic loading. Technical Report MCEER-07-0012, Multidisciplinary Center for Earthquake Engineering Research, 2007.

[23] Constantinou MC, Mokha A, Reinhorn AM. Teflon bearings in base isolation. II: modeling. Journal of Structural Engineering 1990; 116(2), pp. 455-474, DOI: 10.1061/(ASCE)0733-9445(1990)116:2(455)

[24] Dolce M, Cardone D, Croatto F. Frictional behaviour of steel-PTFE interfaces for seismic isolation. Bulletin of Earthquake Engineering 2005; 3(1), 75-99, DOI: 10.1007/s10518-005-0187-9.

[25] Quaglini V, Dubini P, Poggi C. Experimental assessment of sliding materials for seismic isolation systems. Bulletin of Earthquake Engineering 2012; 10(2), pp. 717-740, DOI: 10.1007/s10518-011-9308-9.

[26] Quaglini V, Bocciarelli M, Gandelli E, Dubini P. Numerical Assessment of Frictional Heating in Sliding Bearings for Seismic Isolation. Journal of Earthquake Engineering, 2014; 18(8), pp. 1198-1216, DOI: $10.1080 / 13632469.2014 .924890$. 
[27] Lomiento G, Bonessio N, Benzoni G. Friction Model for Sliding Bearings under Seismic Excitation. Journal of Earthquake Engineering 2013; 17(8), pp. 1162-1191, DOI:10.1080/13632469.2013.814611.

[28] Cardone D, Gesualdi G, Brancato P. Restoring capability of friction pendulum seismic isolation systems. Bulletin of Earthquake Engineering 2015; 13(8), pp 2449-2480, DOI: 10.1007/s10518-014-9719-5.

[29] Sarlis AA, Constantinou MC, Reinhorn AM. Shake table testing of Triple Friction Pendulum Isolators under extreme conditions. Technical Report NCEER-13-0011, National Center for Earthquake Engineering Research, 2013.

[30] Al-Hussaini TM, Zayas VA, Constantinou MC. Seismic isolation of a multi-story frame structure using spherical sliding isolation systems. Technical Report No. NCEER-94-0007, National Center for Earthquake Engineering Research, 1994.

[31] Medeot R. Lesson Learned from Shake Table Tests on Flat and Curved Surface Sliders. Proceedings of the Seventh World Congress on Joints, Bearings and Seismic Systems for Concrete Structures, 2011, Las Vegas, USA.

[32] Ismail M, Rodellar J, Pozo F. An isolation device for near-fault ground motions. Structural Control and Health Monitoring 2014; 21(3), pp. 249-268, DOI: 10.1002/stc.1549.

[33] Calabrese A. Spizzuoco MC, Serino G, Della Corte G, Maddaloni G. Shaking table investigation of a novel, lowcost, base isolation technology using recycled rubber. Structural Control and Health Monitoring, 2015; 22(1), pp. 107-122, DOI: $10.1002 /$ stc. 1663 .

[34] Fenz DM, Constantinou MC. Behaviour of the double concave Friction Pendulum bearing. Earthquake Engineering and Structural Dynamics 2006; 35(11), pp. 1403-1424, DOI: 10.1002/eqe.589.

[35] NTC, 2008. Approvazione delle nuove norme tecniche per le costruzioni, Gazzetta Ufficiale della Repubblica Italiana n. 29 del 4 febbraio 2008 - Suppl. Ordinario n. 30, (in Italian).

[36] Ambraseys N, Smit P, Sigbjornsson R, Suhadolc P, Margaris B. http://www.isesd.cv.ic.ac.uk/ESD/ Internet-Site for European Strong-Motion Data, European Commission, Research-Directorate General, Environment and Climate Programme, 2002.

[37] Iervolino I, Galasso C, Cosenza E. REXEL: computer aided record selection for code-based seismic structural analysis. Bulletin of Earthquake Engineering 2010; 8(2), pp. 339-362, DOI: 10.1007/s10518-009-9146-1.

[38] Bozzo S, Harbat AH. Nonlinear response of structures with sliding base isolation. Structural Control and Health Monitoring 1995; 2 (2), pp. 59-77, DOI: 10.1002/stc.4300020203. 
[39] Fagà E, Ceresa P, Nascimbene R, Moratti M, Pavese A. Modelling curved surface sliding bearings with bilinear constitutive law: effects on the response of seismically isolated buildings. Materials and Structures $\mathbf{2 0 1 5}$ (published on line). DOI 10.1617/s11527-015-0642-2.

[40] Roussis PC, Constantinou MC. Experimental and analytical studies of structures seismically isolated with an uplift-restraining friction pendulum system. Earthquake Engineering \& Structural Dynamics 2006; 35, pp. 595611. DOI: $10.1002 /$ eqe.546.

[41] Ryan K, Dao N. Influence of vertical ground shaking on horizontal response of seismically isolated buildings with friction bearings. Journal of Structural Engineering 2016; 142(1), DOI: 10.1061/(ASCE)ST.1943-541X.0001352, 04015089.

[42] Mosqueda G, Whittaker AS, Fenves GL. Characterization and modeling of friction pendulum bearings subjected to multiple components of excitation. Journal of Structural Engineering 2004; 130(3), pp. 433-442, DOI: 10.1061/(ASCE)0733-9445(2004)130:3(433).

[43] Morgan TA, Mahin SA. The use of innovative base isolation systems to achieve complex seismic performance objectives. Technical Report No. PEER-2011/06, Pacific Earthquake Engineering Research Center, 2011,

[44] Fenz DM, Constantinou MC. Development, implementation and verification of dynamic analysis models for multi-spherical sliding bearings. Technical Report No, MCEER-08-0018, Multidisciplinary Center for Earthquake Engineering Research, NY, 2008.

[45] Ogawa N, Ohtani K, Katayama T, Shibata H, Construction of a three-dimensional, large-scale shaking table and development of core technology. Philosophical Transactions of the Royal Society A 2001; 359, pp. 1725-1751, DOI: DOI: $10.1098 /$ rsta.2001.0871.

[46] Ohtani K, Ogawa N, Katayama T, Shibata H. Construction of E-Defense (3-D full-scale earthquake testing facility). Proceedings of the 13th World Conference on Earthquake Engineering 2004, paper 189, Vancouver, Canada. 